\title{
A coupled 3D-1D numerical monodomain solver for cardiac electrical activation in the myocardium with detailed Purkinje network
}

\author{
Christian Vergara ${ }^{\mathrm{a}, *}$, Matthias Lange ${ }^{\mathrm{b}}$, Simone Palamara ${ }^{\mathrm{a}}$, Toni Lassila ${ }^{\mathrm{b}}$, \\ Alejandro F. Frangi ${ }^{b}$, Alfio Quarteroni ${ }^{\mathrm{c}}$ \\ a MOX, Dipartimento di Matematica, Politecnico di Milano, Italy \\ b CISTIB, Department of Electronic and Electrical Engineering, The University of Sheffield, United Kingdom \\ c Chair of Modelling and Scientific Computing, École Polytechnique Fédérale de Lausanne, Switzerland
}

\section{A R T I C L E I N F O}

\section{Article history:}

Received 10 April 2015

Received in revised form 22 November 2015

Accepted 7 December 2015

Available online 21 December 2015

\section{Keywords:}

Computational electrocardiology

Purkinje fibers

Monodomain equation

Pull and push effect

\begin{abstract}
A B S T R A C T
We present a model for the electrophysiology in the heart to handle the electrical propagation through the Purkinje system and in the myocardium, with two-way coupling at the Purkinje-muscle junctions. In both the subproblems the monodomain model is considered, whereas at the junctions a resistor element is included that induces an orthodromic propagation delay from the Purkinje network towards the heart muscle. We prove a sufficient condition for convergence of a fixed-point iterative algorithm to the numerical solution of the coupled problem. Numerical comparison of activation patterns is made with two different combinations of models for the coupled Purkinje network/myocardium system, the eikonal/eikonal and the monodomain/monodomain models. Test cases are investigated for both physiological and pathological activation of a model left ventricle. Finally, we prove the reliability of the monodomain/monodomain coupling on a realistic scenario. Our results underlie the importance of using physiologically realistic Purkinje-trees with propagation solved using the monodomain model for simulating cardiac activation.
\end{abstract}

(C) 2015 Elsevier Inc. All rights reserved.

\section{Introduction}

The Purkinje fibers are a dense network of specialized cells located under the inner surface of the heart (the endocardium) and are responsible for the fast conduction of the activation signal from the atrioventricular node to the heart muscle (myocardium). The inclusion of the Purkinje fibers in computational models of electrocardiology has been in recent years recognized as fundamental to accurately describing the electrical activation in the left ventricle [1-7]. These fibers form a network, which represents the peripheral part of the conduction system.

The electrical propagation in the Purkinje fibers has been treated with different mathematical models derived from those commonly used for the electrical propagation in the myocardium. We cite for example the eikonal model [4-6,8], the monodomain model [9,10], and the bidomain model [11,12]. In normal electrical propagation, the electrical signal, originating from the atrioventricular (AV) node, travels along this network and enters the ventricular muscle through the

\footnotetext{
* Correspondence to: Piazza Leonardo da Vinci 32, 20133, Milan, Italy. Tel.: +39 0223994610.

E-mail addresses: christian.vergara@polimi.it (C. Vergara), m.lange@sheffield.ac.uk (M. Lange), simone.palamara@polimi.it (S. Palamara), t.lassila@sheffield.ac.uk (T. Lassila), a.frangi@sheffield.ac.uk (A.F. Frangi), alfio.quarteroni@epfl.ch (A. Quarteroni).
} 
Purkinje-muscle junctions (PMJ). In pathological situations, such as the Wolff-Parkinson-White (WPW) syndrome, the signal may enter the myocardium from different regions so that two fronts propagate at the same time, one from the network towards the myocardium and another one in the opposite direction. Capturing the coupled nature of propagation arising from the interaction between the Purkinje network and the myocardium is a fundamental modeling issue. In this regard, different coupled models have been considered in the literature. We cite the coupled eikonal/eikonal model $[13,5,6,8]$ (the first model refers to the one used for the network, whereas the second to the one used for the myocardium), the eikonal/monodomain model [4], the monodomain/bidomain model [9], the monodomain/monodomain model [10], and the bidomain/bidomain model $[11,12]$. When monodomain or bidomain models are considered, the issue of the coupling between cardiac muscle and Purkinje network should be properly addressed. Indeed, due to the parabolic nature of these models, an explicit algorithm based on the successive solution of the propagation in the network and in the muscle only once per time step could not reproduce accurate results when multiple fronts are propagating (as happens for WPW). The works cited above considered explicit coupling strategies which give accurate results only for a normal propagation.

In this work, we start from the monodomain model proposed for the Purkinje network in [9] and consider the coupling with the monodomain model in the myocardium, obtaining a monodomain/monodomain coupled problem (Sect. 2). We observe that with the monodomain formulation we are not able to study some specific feature of pathological propagations, such as the heart fibrillation. However, this model is able to capture many characteristic features of the electrical propagation in the heart, in particular it is suited in view of the electro-mechanical coupling. Since it allows to highly reduce the computational time with respect to the bidomain model, in this work we chose this simplified model. This allowed us to perform a well-posedness analysis of the coupled problem (difficultly applicable to the bidomain context). Moreover, we introduce a semi-implicit time discretization and an iterative algorithm for the solution of the coupled problem arising at each time step (Sect. 3). We observe that the proposed algorithm allows us to treat implicitly the coupling conditions between Purkinje network and cardiac muscle, thus solving a truly coupled problem which is able to describe also situations where multiple fronts propagate.

Finally, we present several numerical results with the aim of assessing the effectiveness of the proposed algorithm and comparing the solutions with the ones obtained with the eikonal/eikonal model. In particular, we discuss the choice of the conduction velocities in the eikonal and monodomain models required to obtain comparable results (Sect. 4.1). We also perform a comparison for a benchmark test between the eikonal/eikonal and the monodomain/monodomain strategies, highlighting the "pull and push" effect (Sect. 4.2) and then consider both normal and pathological (WPW syndrome) propagations in an ideal ellipsoidal model of the ventricle (Sect. 4.3). Interestingly, with our algorithm we are able to recover, without any a priori imposition, some of the more interesting features of the electrical propagation in the heart, such as the pull and push effect, and the delay at the PMJ. Finally we apply the monodomain/monodomain methodology to a realistic case (Sect. 4.4).

\section{Mathematical models for the electrical activation}

In this section we provide the mathematical models considered in this work for the description of the electrical activation in the myocardium and in the Purkinje network, and the corresponding coupled problem. We will use the subscripts $m$ and $p$ to characterize the quantities related to the myocardium and to the Purkinje network, respectively.

\subsection{Activation in the myocardium}

\subsubsection{Monodomain model in the myocardium}

The bidomain model, which accounts for the propagation of the extra- and intra-cellular potentials (see, e.g., [14-16]), is the most commonly used model to describe the electrical activation in the myocardium. To reduce the high computational costs associated to using the bidomain model, the simpler monodomain model, which describes the evolution of the transmembrane potential $V_{m}$ in the myocardium domain $\Omega_{m}$, is often used. It reads as follows:

Given $V_{m, 0}$ and $\boldsymbol{w}_{m, 0}$, find $V_{m}: \Omega_{m} \times(0, T] \rightarrow \mathbb{R}$ and $\boldsymbol{w}_{m}: \Omega_{m} \times(0, T] \rightarrow \mathbb{R}^{d_{m}}$, such that

$$
\begin{cases}\chi_{m}\left(\mathcal{C}_{m} \frac{\partial V_{m}}{\partial t}+I_{i o n}^{m}\left(V_{m}, \boldsymbol{w}_{m}\right)\right)-\nabla \cdot\left(\Sigma \nabla V_{m}\right)=I & \text { in } \Omega_{m} \times(0, T), \\ \frac{d \boldsymbol{w}_{m}}{d t}=\boldsymbol{f}_{m}\left(V_{m}, \boldsymbol{w}_{m}\right) & \text { in } \Omega_{m} \times(0, T), \\ \left(\Sigma \nabla V_{m}\right) \boldsymbol{n}=0 & \text { on } \partial \Omega_{m} \times(0, T), \\ V_{m}(\boldsymbol{x}, 0)=V_{m, 0}(\boldsymbol{x}), \boldsymbol{w}_{m}(\boldsymbol{x}, 0)=\boldsymbol{w}_{m, 0}(\boldsymbol{x}) & \text { in } \Omega_{m},\end{cases}
$$


where $\Sigma$ is the conductivity tensor given by

$$
\Sigma(\boldsymbol{x})=\sigma_{t} \boldsymbol{I}+\left(\sigma_{f}-\sigma_{t}\right) \boldsymbol{a}_{f}(\boldsymbol{x}) \boldsymbol{a}_{f}(\boldsymbol{x})^{T},
$$

$\sigma_{t}$ and $\sigma_{f}$ are the conductivities in the orthogonal and longitudinal directions with respect to the fibers, and $\boldsymbol{a}_{f}$ is the unit vector aligned with the fibers. $\chi_{m}$ is the surface-to-volume ratio of the cell membrane, $\mathcal{C}_{m}$ is the membrane capacitance, $I_{m}^{\text {ion }}$ represents the ionic currents (more precisely, current densities per surface unit), $\boldsymbol{w}_{m}$ is the unknown vector that includes the gating and ion concentration variables of the ODE system representing a suitable cell model, and the vectorial function $\boldsymbol{f}_{m}$ is a non-linear term which determines the evolution of $\boldsymbol{w}_{m}$. We have considered the no-flux boundary condition (1) 3 on the ventricle [1]. The forcing term I, representing an external current (more precisely, a current density per volume unit), will be specified once we couple this system with the 1D monodomain one, see Section 2.3.1.

For the sake of exposition, in what follows we will compactly write problem (1) as follows

$$
P_{m}\left(V_{m}, \boldsymbol{w}_{m}, I\right)=0 .
$$

The monodomain model is based on the assumption of equal anisotropy ratio between the intra- and extra-cellular domains. If there is no injection of current into the extracellular domain, this model is indeed a good approximation of the more complex bidomain one [17,18]. Notice that (1) is a coupled problem, since the transmembrane potential and the gating/ion concentration variables appear in both the differential problems through the coupling terms $I_{m}^{\text {ion }}$ and $\boldsymbol{f}_{m}$.

\subsubsection{Eikonal model in the myocardium}

If one is interested only in the ventricular activation times, defined as the time at which the potential reaches the intermediate value between the maximum and the resting potential [19,20], then a further simplified model could be considered, namely the eikonal model, that provides at each point the activation time. This model discards all the cellular kinetics and describes only the macroscopic spreading of the excitation wavefronts. As such, it does not require a fine spatial resolution, making it possible to simulate the activation of large volumes of cardiac tissue at low computational costs. It is indeed a good approximation of the bidomain model [21] for the computation of activation times, whereas it is unsuitable to describe re-entrant phenomena such as arrhythmias.

In this work we consider the anisotropic eikonal equation, which reads:

Given $u_{m, 0}$, find the activation times $u_{m}: \Omega_{m} \rightarrow \mathbb{R}$ such that

$$
\begin{cases}C_{f} \sqrt{\left(\nabla u_{m}\right)^{T} D \nabla u_{m}}=1 & \boldsymbol{x} \in \Omega_{m}, \\ u_{m}(\boldsymbol{x})=u_{m, 0}(\boldsymbol{x}) & \boldsymbol{x} \in \Gamma_{m},\end{cases}
$$

where $\Gamma_{m}$ is the set of boundary points generating the front, $D(\boldsymbol{x})$ models the anisotropic tensor that accounts for the presence of the muscular fibers, and $C_{f}(\boldsymbol{x})$ represents the velocity of the depolarization wave along the fiber direction. We use the following expression [20]

$$
D(\boldsymbol{x})=k^{2} \boldsymbol{I}+\left(1-k^{2}\right) \boldsymbol{a}_{f}(\boldsymbol{x}) \boldsymbol{a}_{f}(\boldsymbol{x})^{T},
$$

where $k$ is the ratio between the conduction velocities in the orthogonal and longitudinal directions with respect to the fibers.

Note that since we did not consider any diffusive term in the eikonal problem, our model does not take into account the effects of wavefront curvature or the interaction between a wavefront with either the domain boundaries or with other fronts. This is justified by observing that in our case the myocardial activation is regulated by the Purkinje fibers, and because of their high density, the diffusion term gives a small contribution with respect to the advection one.

Problem (2) can be solved very efficiently by the fast marching method [22] and has been successfully used for clinical applications, see $[13,5,6]$.

\subsection{Activation in the Purkinje network}

\subsubsection{Monodomain model in the network}

Both the cardiomyocytes in the myocardium and the ones in the Purkinje network are electrically connected by gapjunctions, intercellular channels providing a low resistance pathway for the spreading of the action potential [23]. Unlike what is usually done in the myocardium where the effect of the gap-junctions, as a consequence of the homogenization process, is hidden in the conductivity tensor $D$, in [9] the authors proposed to explicitly model a gap-junctions in the Purkinje network as a resistor placed between two Purkinje cells. This allows us to easily write the Kirchhoff laws at the bifurcation points of the network, since the potential and the current in the Purkinje cell/gap junction unit are treated as independent variables. Moreover, as highlighted in [9], this "discrete" approach, in antithesis to a homogenized one, allows one to describe the sawtooth effect, see [9] for more details. 


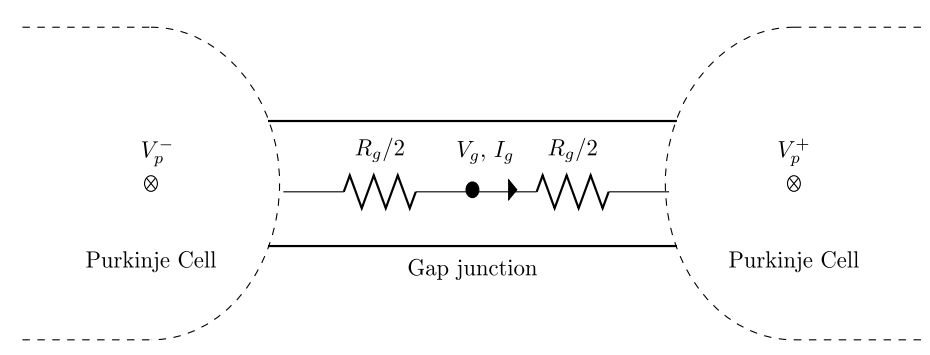

Fig. 1. Schematic representation of a gap-junction linking two Purkinje cells.

In this work we use the discrete model proposed in [9]. As a consequence, the gap-junction resistance needs to be compatible with the homogenized conduction tensor of a single Purkinje cell/gap junction unit. To this aim, let $\sigma_{p}^{*}$ denote the conductivity in the cells, and $R_{g}$ the resistance over the gap-junction. Both together determine the equivalent conductivity $\sigma_{p}=\left(\sigma_{p}^{*} l\right) /\left(l+\sigma_{p}^{*} R_{g} \pi \rho^{2}\right)$ of a single cell/gap-junction unit, where $l$ is the length of the cell and $\rho$ its radius. It is important to note that, in contrast to [9], this choice of the equivalent conductivity depends on the physical properties of the cell and not on the numerical parameters (space discretization step).

We therefore have a sequence of elementary units composed by two Purkinje cells connected by a gap junction, which are characterized by the same spatial coordinates (see Fig. 1). Each of these units is characterized by the extra-cellular and intra-cellular potentials and by the currents related to the cells at the left and at the right (identified with the index - and + , respectively) and to the gap junction (identified with the index $g$ ). We assume here that the extra-cellular potential $\phi_{e}$ is constant for each unit, so that we can consider the transmembrane potential as the effective potential unknown. Thus, for each unit the unknowns of the problem are the transmembrane potentials $V_{g}, V_{p}^{+}, V_{p}^{-}$and the currents $I_{g}, I_{p}^{+}, I_{p}^{-}$.

We assume that the bifurcation and intersection points of the network are located in correspondence of some of the gap-junctions. Kirchhoff laws at the generic bifurcation or intersection yield

$$
\sum_{j=1}^{p} I_{g, j}=0, \quad V_{g, 1}=\ldots=V_{g, p}
$$

where $p$ is the number of branches issuing from the bifurcation or intersection and we adopt the usual convention that entering currents are positive and exiting currents are negative.

From Ohm's law at the gap-junctions, we also have

$$
I_{g}= \pm \frac{V_{g}-V_{p}^{ \pm}}{R_{g} / 2}
$$

The intracellular current $I_{p}^{ \pm}$that flows in the Purkinje cell can be written as

$$
I_{p}^{ \pm}=-\pi \rho^{2} \sigma_{p} \frac{\partial V_{p}^{ \pm}}{\partial l}
$$

where $\rho$ is the radius of the Purkinje cell, and $\sigma_{p}$ is the equivalent intracellular conductivity [9]. Thanks to the conservation of currents at the gap-junction, $I_{g}=I^{+}=I^{-}$, we have

$$
I_{g}=-\pi \rho^{2} \sigma_{p} \frac{\partial V_{p}^{+}}{\partial l}=\pi \rho^{2} \sigma_{p} \frac{\partial V_{p}^{-}}{\partial l}
$$

To summarize, the monodomain model with gap-junctions in the Purkinje network is given by the monodomain equation written in each segment of the network, together with the relations at the gap-junctions (5)-(6) and with the continuity relations at the bifurcation/intersection points (4):

Given $V_{p, 0}, \boldsymbol{w}_{p, 0}$ and $h_{A V}$, find $V_{p, i}^{ \pm}: S_{i} \times(0, T] \rightarrow \mathbb{R}, V_{g, i}: S_{i} \times(0, T] \rightarrow \mathbb{R}$ and $\boldsymbol{w}_{p, i}: S_{i} \times(0, T] \rightarrow \mathbb{R}^{d_{p}}, i=1, \ldots, P$, such that 


$$
\begin{cases}\chi_{p}\left(\mathcal{C}_{p} \frac{\partial V_{p, i}^{ \pm}}{\partial t}+I_{p}^{i o n}\left(V_{p, i}^{ \pm}, \boldsymbol{w}_{p, i}^{ \pm}\right)\right)-\frac{\partial}{\partial l}\left(\sigma_{p} \frac{\partial V_{p, i}^{ \pm}}{\partial l}\right)=0 & \text { in } S_{i} \times(0, T], \quad i=1, \ldots, P, \\ \frac{\partial \boldsymbol{w}_{p, i}^{ \pm}}{\partial t}+\boldsymbol{f}_{p}\left(V_{p, i}^{ \pm}, \boldsymbol{w}_{p, i}^{ \pm}\right)=\mathbf{0} & \text { in } S_{i} \times(0, T], \quad i=1, \ldots, P, \\ V_{g, i}=V_{p, i}^{+}+\frac{I_{g, i} R_{g}}{2}=V_{p, i}^{-}-\frac{I_{g, i} R_{g}}{2} & \text { in } S_{i} \times(0, T], \quad i=1, \ldots, P, \\ I_{g, i}=-\pi \rho^{2} \sigma_{p} \frac{\partial V_{p, i}^{+}}{\partial l}=\pi \rho^{2} \sigma_{p} \frac{\partial V_{p, i}^{-}}{\partial l} & \text { in } S_{i} \times(0, T], \quad i=1, \ldots, P, \\ \sum_{i=i_{1}^{i}{ }_{p_{k}}^{k} I_{g, i}=0} & \text { at } \boldsymbol{b}_{k}, k=1, \ldots, P, t \in(0, T], \\ V_{g, i_{1}^{k}}=\ldots=V_{g, i_{p_{k}}^{k}} & \text { at } \boldsymbol{b}_{k}, k=1, \ldots, P, t \in(0, T], \\ -\pi \sigma_{p} \rho^{2} \frac{\partial V_{p}^{ \pm}}{\partial l}\left(\boldsymbol{s}_{0}\right)=h_{A V} & t \in(0, T], \\ -\pi \sigma_{p} \rho^{2} \frac{\partial V_{p}^{ \pm}}{\partial l}\left(\boldsymbol{s}_{j}\right)=N_{j} & j=1, \ldots, N, t \in(0, T], \\ V_{p}^{ \pm}=V_{p, 0}(x) & \text { in } \Omega_{p}, \\ \boldsymbol{w}_{p}^{ \pm}=\boldsymbol{w}_{p, 0}(x) & \text { in } \Omega_{p},\end{cases}
$$

where $S_{i}$ are the segments of the network such that $\bigcup_{i=1}^{p} S_{i}=\Omega_{p}, \Omega_{p}$ being the Purkinje network domain, $\chi_{p}$ the surfaceto-volume ratio of the cell membrane, $I_{p}^{\text {ion }}$ the ionic currents (or current densities per surface unit), $\mathcal{C}_{p}(\boldsymbol{x})$ is the membrane capacitance, $l$ is the curvilinear coordinate along the network, $\boldsymbol{s}_{0}$ the coordinate of the atrioventricular node, $\boldsymbol{s}_{j}, j=1, \ldots, N$, the coordinates of the PMJ, $\boldsymbol{b}_{k}$ the coordinates of the bifurcation and intersection points, and $i_{1}^{k}, \ldots, i_{p_{k}}^{k}$ are the $p_{k}$ indices related to the potentials and currents involved at the bifurcation/intersection point $\boldsymbol{b}_{k}$. Equations (7) 8 represent Neumann boundary conditions at the PMJ, which are either inlets or outlets for the system. We leave for the moment the data $N_{j}$ unspecified: they will be provided by the coupling with the myocardial activation, see Sect. 2.3.1.

For the sake of exposition, in what follows we will compactly write problem (7) as follows

$$
P_{p}\left(V_{p}^{+}, V_{p}^{-}, V_{g}, I_{g}, \boldsymbol{w}_{p}^{+}, \boldsymbol{w}_{p}^{-}, \boldsymbol{N}\right)=0,
$$

where the unknowns are defined globally in all the network starting from their value on each segment $S_{i}$.

A computational convergence analysis of the numerical solution towards the exact one for problem (7) in the Purkinje network has been performed by us in [24]. This is the first attempt to validate the fully discrete representation of the network given by gap-junction/Purkinje cell units. The results showed convergence of the solution both for steady and pulsatile test cases.

\subsubsection{Eikonal model in the network}

In the case of a network of one-dimensional line segments representing the Purkinje fibers, we can consider again the eikonal model without diffusion:

Given $u_{p, 0}$, find the activation times $u_{p}: \Omega_{p} \rightarrow \mathbb{R}$ such that

$$
\begin{cases}C_{p}\left|\frac{\partial u_{p}}{\partial l}\right|=1 & \boldsymbol{x} \in \Omega_{p}, \\ u_{p}(\boldsymbol{x})=u_{p, 0}(\boldsymbol{x}) & \boldsymbol{x} \in \Gamma_{p},\end{cases}
$$

where $\Gamma_{p}$ is the set of points generating the front in the network (for example, in a normal propagation, the AV node) and $C_{p}$ the conduction velocity (5-10 times greater than the muscular one [25]). Again, we neglect the diffusion term since the high advection term $V_{p}$ dominates any diffusion process.

\subsection{Coupled problems}

The Purkinje fibers form a subendocardial network characterized by a high conduction velocity and are isolated from the muscle, except at their endpoints, the PMJ, which are located on the endocardium. Through the PMJ, the signal could either enter the ventricle from the network, as in a normal propagation (orthodromic propagation), or enter the network from the myocardium, as happens for some pathological conditions (antidromic propagation), see, e.g. [6]. In both cases a delay at the PMJ is observed, in particular an orthodromic delay $d_{o}$ of about 5-15 ms and an antidromic delay $d_{a}$ of about 2-3 ms $[26,27]$. Thus, we have a coupled problem between the electrical propagation in the 1D network and in the 3D myocardium where the coupling points are the PMJ.

In what follows, we describe two possible coupled strategies, namely the monodomain/monodomain (MM) and the eikonal/eikonal (EE) ones. 


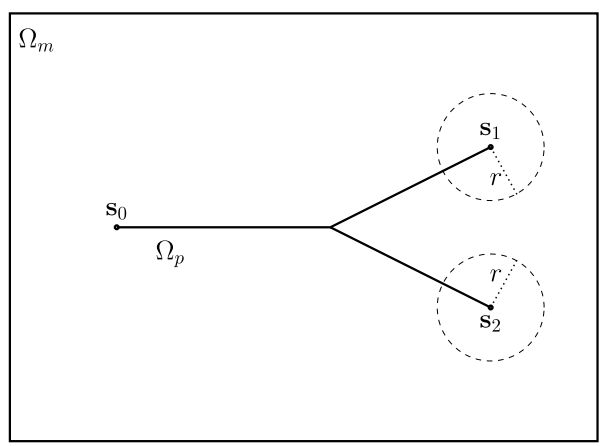

Fig. 2. Schematic representation of a generic myocardial domain $\Omega_{m}$ and of a generic network $\Omega_{p}$. The node $\boldsymbol{s}_{0}$ represents the AV node, whereas the node $\boldsymbol{s}_{1}$ and $\boldsymbol{s}_{2}$ are the PMJ, which act as source terms for the myocardium through the spheres of radius $r$ centered in the PMJ.

\subsubsection{Monodomain/monodomain coupling}

The MM strategy has been introduced in [9], and is based on using (1) for the myocardium and (7) for the Purkinje network. However, in that work the authors considered an explicit coupling between the two subproblems which was based on their sequential solution (network first and then myocardium) only once per time step. This did not allowed the authors to treat cases where, besides the front propagating from the AV node, other fronts originate from the muscle as in pathological conditions. One of the major novelties of the present work is to consider an implicit coupling between the two subproblems, as detailed in what follows.

To write the coupled system, we need to introduce a model describing the propagation of the electrical signal through the PMJ. From histological inspection, PMJs appear to be composed by transitional cells connecting together the distal part of the Purkinje fibers and the surrounding myocardial cells [28]. A detailed model of the PMJ is presented in [29], with the aim of studying the conduction delay at the PMJ. However, in this work we consider a simpler model, based on the introduction of a PMJ resistance [11,9], which provides a good approximation of the real behavior of the PMJ as shown in [30]. The influence of the PMJ on the two subdomains (the myocardium and the Purkinje network) has been modeled in terms of exchange of currents. On one hand, the PMJs act as sources for the myocardium through regions of influence modeled as spheres of radius $r$ centered in the PMJ for a suitable $r$ (see Fig. 2) [11]. On the other hand, the PMJs provide the current to the network through the prescription of Neumann boundary conditions for problem (7) (remember relation $\left.(7)_{4}\right)$.

As discussed, the PMJ has been modeled as a resistance element, so that the current $\gamma_{j}$ at the $j$-th PMJ can be written thanks to the Ohm's law as follows

$$
\gamma_{j}=\frac{\frac{V_{p}^{+}\left(\boldsymbol{s}_{j}\right)+V_{p}^{-}\left(\boldsymbol{s}_{j}\right)}{2}-\frac{1}{A_{r}} \int_{\mathcal{B}_{r}\left(\boldsymbol{s}_{j}\right)} V_{m} d \boldsymbol{x}}{R_{P M J}} j=1, \ldots, N, t \in(0, T],
$$

where $\mathcal{B}_{r}\left(\boldsymbol{s}_{j}\right)$ is the ball of radius $r$ centered at the point $\boldsymbol{s}_{j}, A_{r}$ the volume of this ball and $R_{P M J}$ the resistance of the PMJ (supposed to be the same for all the PMJ). The potential appearing at the numerator of the previous equation is nothing but the jump between the Purkinje network potential and the myocardial potential at the PMJ. Notice that the value of the potential from the Purkinje network side have been chosen as the average of the two potentials $V_{p}^{+}$and $V_{p}^{-}$at the terminal node of the network (the PMJ), since also here we have used a gap-junction model. Instead, the potential from the myocardium side has been computed as the average of the myocardial potential $V_{m}$ over the ball involved in the exchange of the current.

Summarizing, by using the notation introduced in the previous subsections, the coupled MM problem reads as follows:

Find for each $t, V_{p}^{+}, V_{p}^{-}, V_{g}, V_{m}, I_{g}, \boldsymbol{w}_{p}^{+}, \boldsymbol{w}_{p}^{-}, \boldsymbol{w}_{m}^{+} \boldsymbol{w}_{m}^{-}$and $\gamma_{j}, j=1, \ldots, N$, such that

$$
\left\{\begin{aligned}
P_{m}\left(V_{m}, \boldsymbol{w}_{m}, \sum_{j=1}^{N} \frac{1}{A_{r}} \mathcal{I}_{\mathcal{B}_{r}\left(\boldsymbol{s}_{j}\right)} \gamma_{j}+I^{\text {ext }}\right) & =0 \\
P_{p}\left(V_{p}^{+}, V_{p}^{-}, V_{g}, I_{g}, \boldsymbol{w}_{p}^{+}, \boldsymbol{w}_{p}^{-}, \boldsymbol{\gamma}\right) & =0 \\
P_{\text {PMJ }}\left(V_{p}^{+}, V_{p}^{-}, V_{m}, \boldsymbol{\gamma}\right) & =0
\end{aligned}\right.
$$

where $P_{P M J}=0$ represents relations (9), $\mathcal{I}_{Y}$ is the characteristic function related to the region $Y \subset \Omega_{m}$, and $I^{\text {ext }}$ an external current.

\subsubsection{Eikonal/eikonal coupling}

A different strategy consists in coupling the eikonal problems (2) and (8) (EE strategy). Again the coupling is provided at the PMJ, so that the set $\Gamma_{m}$ in (2) and $\Gamma_{p}$ in (8) could contain also some of the PMJ. 
Unlike the MM strategy, in this case it was necessary to identify the orthodromic PMJs, that is the ones that bring the signal from the network to the myocardium, and the antidromic PMJs that bring the signal from the myocardium to the network. Indeed, the solutions of the eikonal problems represent fronts propagating from their source points. Then, in our case we had in general two fronts, one coming from the AV node and another one generated in the myocardium due to pathological conditions (such as the WPW syndrome or the left bundle branch block). We refer the reader to [6,8] for further details.

\section{Numerical solution of the monodomain/monodomain coupled problem}

In this section we propose an algorithm for the numerical solution of the MM coupled problem (10). In particular, in Section 3.1 we first introduce the time discretization followed by a fixed point algorithm, whose convergence analysis is carried out in Section 3.2. Finally, in Section 3.3 we provide details about the numerical solution of the monodomain subproblems arising at each iteration of the fixed point algorithm.

For the numerical solution of the EE coupled problem we adopt here the strategy proposed in [8] for a normal propagation, and extended to treat also pathological conditions in [6]. We refer the reader to these works for further details.

\subsection{Numerical algorithm}

For the 3D problem $(1)_{1}$ we propose a semi-implicit time discretization, with the diffusive term treated implicitly through the backward Euler method, and the coupling term $I_{i o n}^{m}$ treated explicitly. The equation $(1)_{2}$ is discretized with the forward Euler method:

$$
\begin{cases}\frac{\chi_{m} \mathcal{C}_{m}}{\Delta t} V_{m}-\nabla \cdot\left(\Sigma \nabla V_{m}\right)=\frac{\chi_{m} \mathcal{C}_{m}}{\Delta t} V_{m}^{n}-\chi_{m} I_{i o n}^{m}\left(V_{m}^{n}, \boldsymbol{w}_{m}^{n}\right)+I & \text { in } \Omega_{m}, \\ \boldsymbol{w}_{m}=\boldsymbol{w}_{m}^{n}-\Delta t \boldsymbol{f}_{m}\left(V_{m}^{n}, \boldsymbol{w}_{m}^{n}\right) & \text { in } \Omega_{m},\end{cases}
$$

where we have dropped the current index $n+1$ in the unknowns on the left hand side for the sake of simplicity.

The same approach was considered for the time discretization of the $1 \mathrm{D}$ problems $(7)_{1}$ and $(7)_{2}$ :

$$
\begin{cases}\frac{\chi_{p} \mathcal{C}_{p}}{\Delta t} V_{p, i}^{ \pm}-\frac{\partial}{\partial l}\left(\sigma_{p} \frac{\partial V_{p, i}^{ \pm}}{\partial l}\right)=\frac{\chi_{p} \mathcal{C}_{p}}{\Delta t}\left(V_{p, i}^{ \pm}\right)^{n}-\chi_{p} I_{p}^{i o n}\left(\left(V_{p, i}^{ \pm}\right)^{n},\left(\boldsymbol{w}_{p}^{ \pm}\right)^{n}\right) & \text { in } S_{i}, \quad i=1, \ldots, P, \\ \boldsymbol{w}_{p, i}^{ \pm}=\left(\boldsymbol{w}_{p, i}^{ \pm}\right)^{n}-\Delta t \boldsymbol{f}_{p}\left(\left(V_{p, i}^{ \pm}\right)^{n},\left(\boldsymbol{w}_{p, i}^{ \pm}\right)^{n}\right) & \text { in } S_{i}, \quad i=1, \ldots, P .\end{cases}
$$

With this in mind, we can introduce suitable operators $\widetilde{P}_{m}$ and $\widetilde{P}_{p}$ and compactly write the discretized-in-time problems (11) and (12) as $\widetilde{P}_{m}\left(V_{m}, I\right)=0$ and $\widetilde{P}_{p}\left(V_{p}^{+}, V_{p}^{-}, V_{g}, \boldsymbol{N}\right)=0$, respectively $(\boldsymbol{N}$ is again the Neumann data prescribed at the PMJ). Notice that we did not explicitly indicate the dependence of the previous operators on $\boldsymbol{w}_{m}, \boldsymbol{w}_{p}^{+}, \boldsymbol{w}_{p}^{-}$since they are not involved directly in the coupling, and that the dependence of $\widetilde{P}_{p}$ and $\widetilde{P}_{m}$ on the quantities at previous time step is understood. This allows us to write the discretized-in-time version of the MM problem (10) as follows:

Find for each $n, V_{p}^{+}, V_{p}^{-}, V_{g}, V_{m}, I_{g}$ and $\gamma_{j}, j=1, \ldots, N$, such that

$$
\left\{\begin{aligned}
\widetilde{P}_{m}\left(V_{m}, \sum_{j=1}^{N} \frac{1}{A_{r}} \mathcal{I}_{\mathcal{B}_{r}\left(\boldsymbol{s}_{j}\right)} \gamma_{j}+I^{e x t}\right) & =0, \\
\widetilde{P}_{p}\left(V_{p}^{+}, V_{p}^{-}, V_{g}, \boldsymbol{\gamma}\right) & =0, \\
P_{P M J}\left(V_{p}^{+}, V_{p}^{-}, V_{m}, \boldsymbol{\gamma}\right) & =0 .
\end{aligned}\right.
$$

For the solution of the discretized-in-time MM coupled problem (13) we propose a fixed point strategy, where at each iteration the currents $\gamma_{j}$ computed at the previous iteration are used to solve the 3D and the 1D problems, and then the values of the potentials are used to update the PMJ currents. This idea is summarized in Algorithm 1 reported below.

\subsection{Analysis}

In this section we provide a convergence analysis of Algorithm 1 in the particular case of $\Omega_{p}$ composed by a single branch, thus with no bifurcations/intersections. Thanks to the time discretization used, at each time step we obtain a linear problem, hence we can restrict ourselves to analyze the convergence towards the null solution in the case of vanishing forcing terms. We can therefore set $I^{e x t}=0, h_{A V}=0$, and null initial conditions, and set to zero the quantities at the previous time step. Moreover, we notice that since we do not have any bifurcation/intersection points, there are no $V_{g}$ and $I_{g}$ in this case and we have only $V_{p}$ to describe the cell potential, instead of $V_{p}^{+}$and $V_{p}^{-}$. Finally, we can translate the solution $V_{p}$ and $V_{m}$ into $V_{p}=0$ and $V_{m}=0$ corresponding to the resting potential conditions. Moreover, we assume that the ionic currents are zero when the transmembrane potential equals the resting potential. In view of the analysis, we introduce the weak formulations of the monodomain problems. Thus, our fixed point strategy can be rewritten as reported in Algorithm 2. 
Algorithm 1 Solution of the discretized-in-time MM coupled problem.

$$
\frac{\left(V_{p}^{+}\right)^{n}\left(\boldsymbol{s}_{j}\right)+\left(V_{p}^{-}\right)^{n}\left(\boldsymbol{s}_{j}\right)}{2}-\frac{1}{A_{r}} \int_{\mathcal{B}_{r}\left(\boldsymbol{s}_{j}\right)} V_{m}^{n} d \boldsymbol{x}
$$

Let $k$ be the iteration index within each time step. Set $k=0$ and $\gamma_{j}^{(0)}=\gamma_{0, j}:=\frac{\mathcal{B}_{r}\left(\boldsymbol{s}_{j}\right)}{R_{P M J}}, j=1, \ldots, N$, with $\left(V_{p}^{+}\right)^{n},\left(V_{p}^{-}\right)^{n}, V_{m}^{n}$ the converged solution at the previous time step, and choose a tolerance $\varepsilon>0$; then at iteration $k+1$

while $\left(\left\|\boldsymbol{\gamma}^{(k)}-\boldsymbol{\gamma}^{(k-1)}\right\|>\varepsilon\right)$

1. Solve the discretized-in-time monodomain problem (1) in the myocardium with applied currents given by $\boldsymbol{\gamma}^{(k)}$, that is

$$
\widetilde{P}_{m}\left(V_{m}^{(k+1)}, \sum_{j=1}^{N} \frac{1}{A_{r}} \mathcal{I}_{\mathcal{B}_{r}\left(\boldsymbol{s}_{j}\right)} \gamma_{j}^{(k)}+I^{\text {ext }}\right)=0
$$

2. Solve the discretized-in-time monodomain problem (7) in the Purkinje network with Neumann boundary conditions at the PMJ given by $\boldsymbol{\gamma}^{(k)}$, that is

$$
\widetilde{P}_{p}\left(\left(V_{p}^{+}\right)^{(k+1)},\left(V_{p}^{-}\right)^{(k+1)}, V_{g}^{(k+1)}, \boldsymbol{\gamma}^{(k)}\right)=0 ;
$$

3. Compute

$$
\boldsymbol{\gamma}_{j}^{(k+1)}=\frac{\frac{\left(V_{p}^{+}\right)^{(k+1)}\left(\boldsymbol{s}_{j}\right)+\left(V_{p}^{-}\right)^{(k+1)}\left(\boldsymbol{s}_{j}\right)}{2}-\frac{1}{A_{r}} \int_{\mathcal{B}_{r}\left(\boldsymbol{s}_{j}\right)} V_{m}^{(k+1)} d \boldsymbol{x}}{R_{P M J}}, j=1, \ldots, N ;
$$

4. Set $k=k+1$.

end

Algorithm 2 Solution of the reduced MM discretized-in-time coupled problem.

Let $k$ be the iteration index within each time step. Set $k=0$ and $\boldsymbol{\gamma}^{(0)}=\boldsymbol{\gamma}_{0}$, and choose a tolerance $\varepsilon>0$; then at iteration $k+1$

while $\left(\left\|\boldsymbol{\gamma}^{(k)}-\boldsymbol{\gamma}^{(k-1)}\right\|>\varepsilon\right)$

1. Solve the following discretized-in-time monodomain problem in the myocardium with applied currents given by $\boldsymbol{\gamma}^{(k)}$ :

Find $V_{m} \in H^{1}\left(\Omega_{m}\right)$ such that

$$
\int_{\Omega_{m}} \chi_{m} \mathcal{C}_{m} \frac{V_{m}^{(k+1)}}{\Delta t} W_{m} d \boldsymbol{x}+\int_{\Omega_{m}} D \nabla V_{m}^{(k+1)} \cdot \nabla W_{m} d \boldsymbol{x}=\sum_{j=1}^{N} \frac{1}{A_{r}} \int_{\mathcal{B}_{r}\left(\boldsymbol{s}_{j}\right)} \gamma_{j}^{(k)} W_{m} d \boldsymbol{x},
$$

for all $W_{m} \in H^{1}\left(\Omega_{m}\right)$;

2. Solve the following discretized-in-time monodomain problem in the Purkinje network with Neumann boundary conditions at the PMJ given by $\boldsymbol{\gamma}^{(k)}$ :

Find $V_{p} \in H^{1}\left(\Omega_{p}\right)$ such that

$$
\int_{\Omega_{p}} \chi_{p} \mathcal{C}_{p} \frac{V_{p}^{(k+1)}}{\Delta t} W_{p} d l+\int_{\Omega_{p}} \sigma_{p} \frac{\partial V_{p}^{(k+1)}}{\partial l} \frac{\partial W_{p}}{\partial l} d l=-\frac{1}{\pi \rho^{2}} \sum_{j=1}^{N} \gamma_{j}^{(k)} W_{p}\left(\boldsymbol{s}_{j}\right),
$$

for all $W_{p} \in H^{1}\left(\Omega_{p}\right)$;

3. Compute the value of $\boldsymbol{\gamma}_{j}^{(k+1)}$ with (14);

4. Set $k=k+1$.

end

The coupled problem in Algorithm 2 can be rewritten as follows

$$
\begin{cases}V_{m}^{(k+1)}=F_{m}\left(\boldsymbol{\gamma}^{(k)}\right) & \text { in } \Omega_{m}, \\ V_{p}^{(k+1)}=F_{p}\left(\boldsymbol{\gamma}^{(k)}\right) & \text { in } \Omega_{p}, \\ \boldsymbol{\gamma}^{(k+1)}=\boldsymbol{F}_{P M J}\left(V_{p}^{(k+1)}, V_{m}^{(k+1)}\right), & \end{cases}
$$

where $F_{m}: \mathbb{R}^{N} \rightarrow H^{1}\left(\Omega_{m}\right), F_{p}: \mathbb{R}^{N} \rightarrow H^{1}\left(\Omega_{p}\right)$, and $\boldsymbol{F}_{P M J}: H^{1}\left(\Omega_{m}\right) \times H^{1}\left(\Omega_{p}\right) \rightarrow \mathbb{R}^{N}$ provide the explicit expressions of the unknowns obtained from (15), (16) and (14). Algorithm 2 can be written in compact form as the following fixed point iteration

$$
\boldsymbol{\gamma}^{(k+1)}=\mathcal{F}\left(\boldsymbol{\gamma}^{(k)}\right)
$$


where

$$
\mathcal{F}: \mathbb{R}^{N} \rightarrow \mathbb{R}^{N} \text { s.t. } \mathcal{F}(\boldsymbol{\gamma})=\boldsymbol{F}_{P M J}\left(F_{p}(\boldsymbol{\gamma}), F_{m}(\boldsymbol{\gamma})\right) .
$$

To prove the convergence of the previous iterations, we need to show that there exists a constant $C \in[0,1)$ such that

$$
\left\|\mathcal{F}\left(\boldsymbol{\gamma}^{(k)}\right)\right\| \leq C\left\|\boldsymbol{\gamma}^{(k)}\right\| \quad \forall k,
$$

for each $\boldsymbol{\gamma}^{(0)}$, where $\|\cdot\|$ is the usual Euclidean norm. This is what is proved in the following result.

Proposition 1. Under the following assumptions:

- There exist two constants $0<b<B$ such that

$$
b\|\boldsymbol{\xi}\| \leq \xi^{t} D(\boldsymbol{x}) \xi \leq B\|\boldsymbol{\xi}\|^{2}, \quad \forall \xi \in \mathbb{R}^{2},
$$

for a.e. $\boldsymbol{x} \in \Omega_{m}$;

- The parameters $\sigma_{p}$ and $b$ satisfy

$$
\sigma_{p} \geq \frac{4 N^{3 / 2} C_{T}^{2}}{\pi \rho^{2} R_{P M J}^{2}}, \quad b \geq \frac{4 N^{3 / 2}}{A_{r}^{3 / 2} R_{P M J}^{2}},
$$

where $C_{T}$ is the trace constant for the Sobolev space $H^{1}\left(\Omega_{p}\right)$;

- The time step $\Delta t>0$ is chosen such that

$$
\Delta t \leq \min \left\{\frac{\chi_{p} \mathcal{C}_{p}}{\sigma_{p}} ; \frac{\chi_{m} \mathcal{C}_{m}}{b}\right\}
$$

then, there exists a constant $C \in[0,1)$ such that (17) is satisfied.

Proof. From the definition of $\mathcal{F}$ we can write

$$
\begin{aligned}
\left\|\mathcal{F}\left(\boldsymbol{\gamma}^{(k)}\right)\right\|^{2} & =\left\|\boldsymbol{F}_{\text {PMJ }}\left(V_{m}^{(k+1)}, V_{p}^{(k+1)}\right)\right\|^{2} \\
& =\sum_{j=1}^{N}\left|\frac{V_{p}^{(k+1)}\left(\boldsymbol{s}_{j}\right)-\frac{1}{A_{r}} \int_{\mathcal{B}_{r}\left(\boldsymbol{s}_{j}\right)} V_{m}^{(k+1)} d \boldsymbol{x}}{R_{P M J}}\right|^{2} \\
& \leq \frac{2}{R_{P M J}^{2}} \sum_{j=1}^{N}\left(\left(V_{p}^{(k+1)}\left(\boldsymbol{s}_{j}\right)\right)^{2}+\left(\frac{1}{A_{r}} \int_{\mathcal{B}_{r}\left(\boldsymbol{s}_{j}\right)} V_{m}^{(k+1)} d \boldsymbol{x}\right)^{2}\right),
\end{aligned}
$$

where we used the inequality

$$
(a+b)^{2} \leq 2 a^{2}+2 b^{2} a, b \in \mathbb{R} .
$$

Regarding the first term at the right hand side of (21), we can apply the trace theorem (see [31]). We notice that in our case the boundary of the problem in the network is given by the PMJ $\boldsymbol{s}_{j}$ and by the AV node $\boldsymbol{s}_{0}$, so we have

$$
\sum_{j=1}^{N}\left(V_{p}^{(k+1)}\left(\boldsymbol{s}_{j}\right)\right)^{2} \leq \sum_{j=1}^{N}\left(V_{p}^{(k+1)}\left(\boldsymbol{s}_{j}\right)\right)^{2}+\left(V_{p}^{(k+1)}\left(\boldsymbol{s}_{0}\right)\right)^{2} \leq C_{T}\left\|V_{p}^{(k+1)}\right\|_{H^{1}\left(\Omega_{p}\right)}^{2} .
$$

Regarding the second term at the right hand side of (21), we use the following inequality holding for every bounded domain $\Omega$ and $0 \leq p \leq q \leq \infty$ :

$$
\|z\|_{L^{p}(\Omega)} \leq|\Omega|^{\frac{1}{p}-\frac{1}{q}}\|z\|_{L^{q}(\Omega)},
$$

provided that $z \in L^{q}(\Omega)$ and where $|\Omega|$ is the size of the domain. In our case we set $\Omega=\mathcal{B}_{r}\left(\boldsymbol{s}_{j}\right), p=1, q=2$, so we obtain

$$
\left\|V_{m}^{(k+1)}\right\|_{L^{1}\left(\mathcal{B}_{r}\left(\boldsymbol{s}_{j}\right)\right)}=\int_{\mathcal{B}_{r}\left(\boldsymbol{s}_{j}\right)}\left|V_{m}^{(k+1)}\right| d \boldsymbol{x} \leq \sqrt{A_{r}}\left\|V_{m}^{(k+1)}\right\|_{L^{2}\left(\mathcal{B}_{r}\left(\boldsymbol{s}_{j}\right)\right)} .
$$


Therefore, we have the following estimate for the second term at the right hand side of (21)

$$
\begin{aligned}
\sum_{j=1}^{N}\left(\frac{1}{A_{r}} \int_{\mathcal{B}_{r}\left(\boldsymbol{s}_{j}\right)} V_{m}^{(k+1)} d \boldsymbol{x}\right)^{2} & =\frac{1}{A_{r}^{2}} \sum_{j=1}^{N}\left\|V_{m}^{(k+1)}\right\|_{L^{1}\left(\mathcal{B}_{r}\left(\boldsymbol{s}_{j}\right)\right)}^{2} \\
& \leq \frac{A_{r}}{A_{r}^{2}} \sum_{j=1}^{N}\left\|V_{m}^{(k+1)}\right\|_{L^{2}\left(\mathcal{B}_{r}\left(s_{j}\right)\right)}^{2} \\
& \leq \frac{1}{A_{r}} \sum_{j=1}^{N}\left\|V_{m}^{(k+1)}\right\|_{L^{2}\left(\Omega_{m}\right)}^{2} \\
& =\frac{N}{A_{r}}\left\|V_{m}^{(k+1)}\right\|_{L^{2}\left(\Omega_{m}\right)}^{2} \\
& \leq C_{1}\left\|V_{m}^{(k+1)}\right\|_{H^{1}\left(\Omega_{m}\right)}^{2},
\end{aligned}
$$

with $C_{1}=\frac{N}{A_{r}}$. Then, owing to (22) and (24), (21) reads

$$
\left\|\mathcal{F}\left(\boldsymbol{\gamma}^{(k)}\right)\right\|^{2} \leq \frac{2}{R_{P M J}^{2}}\left(C_{T}\left\|V_{p}^{(k+1)}\right\|_{H^{1}\left(\Omega_{p}\right)}^{2}+C_{1}\left\|V_{m}^{(k+1)}\right\|_{H^{1}\left(\Omega_{m}\right)}^{2}\right) .
$$

Now, we have to find suitable estimates for the right hand side of (25) in terms of $\left\|\boldsymbol{\gamma}^{(k)}\right\|$. To this aim, we take $W_{p}=$ $V_{p}^{(k+1)}$ as a test function in (16) obtaining

$$
\frac{\chi_{p} \mathcal{C}_{p}}{\Delta t}\left\|V_{p}^{(k+1)}\right\|_{L^{2}\left(\Omega_{p}\right)}^{2}+\sigma_{p}\left\|\frac{\partial V_{p}^{(k+1)}}{\partial l}\right\|_{L^{2}\left(\Omega_{p}\right)}^{2}=-\frac{1}{\pi \rho^{2}} \sum_{j=1}^{N} \gamma_{j}^{(k)} V_{p}^{(k+1)}\left(\boldsymbol{s}_{j}\right) \text {. }
$$

Thus, we have

$$
C_{2}\left\|V_{p}^{(k+1)}\right\|_{H^{1}\left(\Omega_{p}\right)}^{2} \leq \frac{N C_{T}}{\pi \rho^{2}}\left\|V_{p}^{(k+1)}\right\|_{H^{1}\left(\Omega_{p}\right)} \sum_{j=1}^{N}\left|\gamma_{j}^{(k)}\right|
$$

with $C_{2}=\min \left\{\frac{\chi_{p} \mathcal{C}_{p}}{\Delta t} ; \sigma_{p}\right\}$, and then

$$
\left\|V_{p}^{(k+1)}\right\|_{H^{1}\left(\Omega_{p}\right)} \leq C_{3}\left\|\boldsymbol{\gamma}^{(k)}\right\|,
$$

with $C_{3}=\frac{N^{3 / 2} C_{T}}{C_{2} \pi \rho^{2}}$.

We proceed now by considering the equation in the myocardium (15), and we take $W_{m}=V_{m}^{(k+1)}$ as a test function, obtaining from (18) the estimate

$$
\frac{\chi_{m} \mathcal{C}_{m}}{\Delta t}\left\|V_{m}^{(k+1)}\right\|_{L^{2}\left(\Omega_{m}\right)}^{2}+b\left\|\nabla V_{m}^{(k+1)}\right\|_{L^{2}\left(\Omega_{m}\right)}^{2} \leq \sum_{j=1}^{N} \frac{1}{A_{r}} \gamma_{j}^{(k)} \int_{\mathcal{B}_{r}\left(s_{j}\right)} V_{m}^{(k+1)} d \boldsymbol{x} .
$$

Then, owing to (23), we have

$$
\begin{aligned}
\sum_{j=1}^{N} \frac{1}{A_{r}} \gamma_{j}^{(k)} \int_{\mathcal{B}_{r}\left(\boldsymbol{s}_{j}\right)} V_{m}^{(k+1)} d \boldsymbol{x} & =\frac{1}{A_{r}} \sum_{j=1}^{N}\left|\gamma_{j}^{(k)}\right|\left\|V_{m}^{(k+1)}\right\|_{L^{1}\left(\mathcal{B}_{r}\left(\boldsymbol{s}_{j}\right)\right)} \\
& \leq \frac{\sqrt{A_{r}}}{A_{r}} \sum_{j=1}^{N}\left|\gamma_{j}^{(k)}\right|\left\|V_{m}^{(k+1)}\right\|_{L^{2}\left(\mathcal{B}_{r}\left(s_{j}\right)\right)} \\
& \leq \sqrt{\frac{1}{A_{r}}} \sum_{j=1}^{N}\left|\gamma_{j}^{(k)}\right|\left\|V_{m}^{(k+1)}\right\|_{L^{2}\left(\Omega_{m}\right)} \\
& =\sqrt{\frac{N}{A_{r}}}\left\|\boldsymbol{\gamma}^{(k)}\right\|\left\|V_{m}^{(k+1)}\right\|_{H^{1}\left(\Omega_{m}\right)} .
\end{aligned}
$$


The previous inequality together with (28) gives

$$
\left\|V_{m}^{(k+1)}\right\|_{H^{1}\left(\Omega_{m}\right)} \leq C_{4}\left\|\boldsymbol{\gamma}^{(k)}\right\|,
$$

with $C_{4}=\sqrt{\frac{N}{A_{r}}} \frac{1}{\min \left\{\frac{\chi m}{\Delta t} ; b\right\}}$.

Thus, putting together (25), (27) and (29), we obtain (17) with

$$
C=\frac{2}{R_{P M J}^{2}}\left(C_{T} C_{3}+C_{1} C_{4}\right)=\frac{2}{R_{P M J}^{2}}\left(\frac{N^{3 / 2} C_{T}^{2}}{\min \left\{\frac{\chi_{p} \mathcal{C}_{p}}{\Delta t} ; \sigma_{p}\right\} \pi \rho^{2}}+\left(\frac{N}{A_{r}}\right)^{3 / 2} \frac{1}{\min \left\{\frac{\chi_{m} \mathcal{C}_{m}}{\Delta t} ; b\right\}}\right) .
$$

Due to (20), we obtain

$$
C=\frac{2}{R_{P M J}^{2}}\left(\frac{N^{3 / 2} C_{T}^{2}}{\sigma_{p} \pi \rho^{2}}+\frac{1}{b}\left(\frac{N}{A_{r}}\right)^{3 / 2}\right),
$$

which is less than one because of (19).

Remark 1. We notice that the assumptions on the parameters $\sigma_{p}$ and $b$ given by (19) depend on the value of the trace constant $C_{T}$, which is not computable for general domains. Therefore we cannot determine explicitly the value of $\sigma_{p}$ and $b$ that guarantee that $\mathcal{F}$ is a contraction. Nevertheless, in all the numerical experiments reported in what follows, we experienced that the proposed algorithm not only converges, but it does so (within machine accuracy) in a finite number of iterations.

Remark 2. The restriction on $\Delta t$ given by (19) should be matched with the one required for stability of the forward Euler methods for the ODE systems $(1)_{2}$ and $(7)_{2}$. Thus, the effective $\Delta t$ is the smaller of these two.

\subsection{Solution of the stand-alone subproblems}

In this section we detail the numerical strategies used to solve the 3D and the 1D monodomain subproblems arising at each iteration of Algorithm 1. For the solution of the 3D subproblem, we consider Lagrangian finite elements and an implicit/explicit method, see [1]. For the solution of the 1D subproblem we follow the methodology presented in [9]. In particular, we assume to have a system of gap-junction/Purkinje cells for each node of the mesh. For each segment of the network $S_{i}$, we know the values of $V_{g, i}^{n}$ and $I_{g, i}^{n}$ at the previous time step $t^{n}$. Then, the numerical scheme to compute $V_{g, i}$ and $I_{g, i}$ for each segment $S_{i}$ at time $t^{n+1}$ can be divided into four steps:

1. Recovering the transmembrane potential $\left(V_{p, i}^{ \pm}\right)^{n}$. By considering $(7)_{3}$, we can recover the value of the transmembrane potential as follows:

$$
\left(V_{p, i}^{ \pm}\right)^{n}=V_{g, i}^{n} \mp \frac{I_{g, i}^{n} R_{g}}{2}
$$

2. Operator splitting - first part. We compute the intermediate potentials $\left(V_{p, i}^{ \pm}\right)^{n+1 / 2}$ as follows:

$$
\mathcal{C}_{p} \frac{\left(V_{p, i}^{ \pm}\right)^{n+1 / 2}-\left(V_{p, i}^{ \pm}\right)^{n}}{\Delta t}=-I_{p}^{i o n}\left(\left(V_{p, i}^{ \pm}\right)^{n},\left(\boldsymbol{w}_{p, i}^{ \pm}\right)^{n}\right)
$$

3. Update of $V_{g}$ and $I_{g}$. We compute the intermediate values $V_{g, i}^{n+1 / 2}$ and $I_{g, i}^{n+1 / 2}$ with the following expressions obtained by manipulating the two equations in $(7)_{3}$ :

$$
I_{g, i}^{n+1 / 2}=\frac{\left(V_{p, i}^{+}\right)^{n+1 / 2}-\left(V_{p, i}^{-}\right)^{n+1 / 2}}{R_{g}}, \quad V_{g, i}^{n+1 / 2}=\frac{\left(V_{p, i}^{+}\right)^{n+1 / 2}+\left(V_{p, i}^{-}\right)^{n+1 / 2}}{2} ;
$$

4. Operator splitting - second part. The second part of the operator splitting should be given by

$$
\chi_{p} \mathcal{C}_{p} \frac{V_{p, i}^{ \pm}-\left(V_{p, i}^{ \pm}\right)^{n+1 / 2}}{\Delta t}-\frac{\partial}{\partial l}\left(\sigma_{p} \frac{\partial V_{p, i}^{ \pm}}{\partial l}\right)=0
$$


Table 1

Parameters used in the numerical experiments, suitable references, and physiological ranges.

\begin{tabular}{|c|c|c|c|c|}
\hline & $\mathrm{E}-\mathrm{E}$ & $\mathrm{M}-\mathrm{M}$ & Ref. & Range \\
\hline$\chi_{m}\left(\mathrm{~cm}^{-1}\right)$ & - & 1400 & [35] & - \\
\hline$\chi_{p}\left(\mathrm{~cm}^{-1}\right)$ & - & 1467 & [36] & - \\
\hline$R_{g}(\mathrm{kOhm})$ & - & 500 & [9] & - \\
\hline$r(\mathrm{~cm})$ & - & 0.06 & {$[11,37]$} & {$[0.01-0.1]$} \\
\hline$\rho(\mathrm{cm})$ & - & 0.0017 & [36] & - \\
\hline$R_{\mathrm{PMJ}}(\mathrm{kOhm})$ & - & 11000 & {$[9,38]$} & [1000-25 000] \\
\hline$\varepsilon$ & - & $10^{-5}$ & - & - \\
\hline$d_{o}(\mathrm{~ms})$ & 5.0 & - & {$[26,27]$} & [5-15] \\
\hline$d_{a}(\mathrm{~ms})$ & 2.0 & - & {$[26,27]$} & {$[2-3]$} \\
\hline$\sigma_{p}\left(\mathrm{kOhm}^{-1} \mathrm{~cm}^{-1}\right)$ & - & 35.0 & {$[25]$} & - \\
\hline$\sigma_{f}\left(\mathrm{kOhm}^{-1} \mathrm{~cm}^{-1}\right)$ & - & 1.334 & [35] & - \\
\hline$\sigma_{t}\left(\mathrm{kOhm}^{-1} \mathrm{~cm}^{-1}\right)$ & - & 0.176 & [35] & - \\
\hline
\end{tabular}

Now, by adding these two equations and by dividing by 2 , we obtain thanks to (31)

$$
\chi_{p} \mathcal{C}_{p} \frac{V_{g, i}-V_{g, i}^{n+1 / 2}}{\Delta t}-\frac{\partial}{\partial l}\left(\sigma_{p} \frac{\partial V_{g, i}}{\partial l}\right)=0 .
$$

As finite element basis to solve the previous problem we use the one-dimensional cubic Hermite basis, so that we can directly recover also the derivative of the potential, which is related to the current (recalling (7)4). Hermite finite elements are suitable for such a purpose as they are based upon solving the potential and its derivative at each node.

Finally, once we have detailed how to compute the values of $V_{g, i}$ and $I_{g, i}$ for each single segment of the network, we need to enforce the Kirchhoff laws (7) $)_{5-6}$ to compute the global $V_{g}$ and $I_{g}$. To this aim, we modify the global finite element matrix associated to the collection of (32) by substituting 1's or 0's in the rows related to bifurcation or intersection points accordingly to $(7)_{5-6}$.

\section{Numerical experiments}

In this section we present several numerical results with the aim of assessing the reliability of Algorithm 1 to solve the MM coupled problem and comparing the results with those obtained with the EE coupled problem. First of all, in Section 4.1 we discuss how to estimate a constant conduction velocity from the coupled monodomain problems to be used in the eikonal ones in view of the forthcoming comparison. After this preliminary step, in Section 4.2 we consider an academic test case with simplified geometries to compare the results obtained with the two different strategies, whereas, in Section 4.3 we apply these strategies to simulate both a normal and a pathological propagation in an ellipsoidal idealized left ventricle. Finally, in Section 4.4, we apply Algorithm 1 to a realistic geometry.

All the numerical results related to the MM problem have been obtained with the parallel Finite Element library LifeV, developed at MOX - Politecnico di Milano, REO/ESTIME - INRIA, CMCS - EPFL, and E(CM) ${ }^{2}$ - Emory University. For the 3D monodomain problem we considered $\mathbb{P}_{1}$ Lagrangian finite elements, whereas for the $1 \mathrm{D}$ problem cubic Hermite finite elements. For both the monodomain problems, we chose a time step $\Delta t=0.01 \mathrm{~ms}$. The ionic models used in our numerical experiments were the Di Francesco-Noble model [32] for the Purkinje cells, and the Luo-Rudy-I model [33] for the myocardial cells. The numerical schemes for solving the coupled EE problem have been implemented in a stand-alone and serial code based on the VTK 5.8.0 library [6,8]. For the solution of the single eikonal problems, we considered the fast marching method (FMM) proposed in [22] for the 1D problem and the modified version of the FMM proposed in [34] for the 3D problem.

If not otherwise specified, in all the numerical experiments we used the data collected in Table 1, where we reported also suitable references and, for some, the ranges of the values reported therein.

Notice that we did not need to prescribe explicitly the delay at the PMJ in the MM model, since in this case the PMJ resistance model itself was able to introduce suitable delays.

\subsection{Assessing the conductivities in view of the comparisons}

In the set-up of the forthcoming numerical tests, we faced two critical points: (i) the choice of proper quantities to be compared in view of a discussion of the results, and (ii) the use of comparable conduction velocities in both the MM and EE coupled problems.

The first issue is crucial because the output of the monodomain problem is the transmembrane potential, whereas the one of the eikonal problem is the local activation time. Then, in view of the comparisons, we computed from the transmembrane potentials the activation times provided by the monodomain problems, defined again as the time at which the potential reaches the mean value between the resting potential and the plateau potential. This allowed us to compare these values with the ones provided by the EE problem. To this aim, we denoted with $u_{p}^{M}(\boldsymbol{x})$ and $u_{m}^{M}(\boldsymbol{x})$ the activation times 


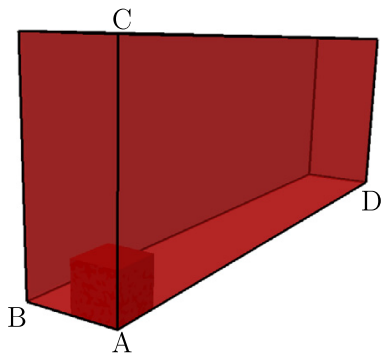

Fig. 3. Myocardial domain. Test for the estimation of the conduction velocity.
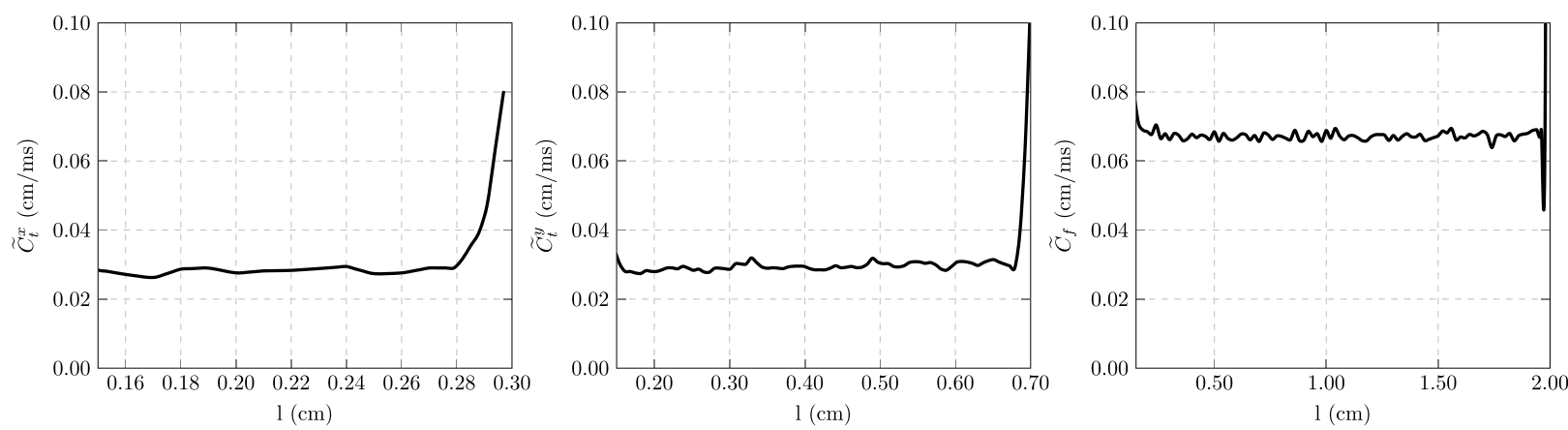

Fig. 4. Conduction velocities in the myocardium as a function of the local coordinate $l$ estimated from the solution of a monodomain problem: $\widetilde{C}_{t}^{x}$ on $\overline{A B}$ (left), $\widetilde{C}_{t}^{y}$ on $\overline{A C}$ (center), and $\widetilde{C}_{f}$ on $\overline{A D}$ (right). Test for the estimation of the conduction velocity.

in the network and in the myocardium, respectively, obtained by solving the MM problem, and with $u_{p}^{E}(\boldsymbol{x})$ and $u_{m}^{E}(\boldsymbol{x})$ the activation times in the network and in the myocardium, respectively, provided by the EE strategy.

For what concerns point (ii) above, we needed to use comparable parameters in order to obtain meaningful results. In particular, we remark that the propagation velocities have a different nature in the monodomain model than in the eikonal one. Indeed, in the first case the conduction velocity of the electrical signal is not constant in time and space and depends on the solution. For example, the propagation velocity changes when two wavefronts collide or when the wavefront interacts with the boundary of the domain. On the contrary, in the eikonal problems, the conduction velocity is a prescribed parameter of the model, and therefore it does not depend on the solution of the problem. However, we observe that in the case of a single wavefront, in the monodomain cases the conduction velocity is almost constant far from the boundaries. This suggests a strategy to estimate a reference constant conduction velocity from the monodomain model, to be then used in the eikonal model. This was done for both the Purkinje network and the myocardium.

To this aim, we considered two reference scenarios, one for the myocardium given by the cuboid with dimensions $0.3 \times 0.7 \times 2.0 \mathrm{~cm}$, see Fig. 3, and one for the network given by a single Purkinje fiber. For the myocardium, we estimated two conduction velocities, one in the direction of the fibers $\left(C_{f}\right)$ which is parallel to $\overline{A D}$, and the other one in the direction transverse to the fiber $\left(C_{t}\right)$. To do this, we solved the monodomain problem in the cuboid with a source current applied in the internal corner of size $0.15 \mathrm{~cm}$ with one of the corners coinciding with $A$ and sides parallel to the ones of the cuboid, see Fig. 3. This allowed to obtain the activation time $u_{m}^{M}(\boldsymbol{x})$ and to define the following velocities

$$
\begin{aligned}
& \widetilde{C}_{f}(\boldsymbol{x}):=\frac{1}{\left|\frac{\partial u_{m}^{M}}{\partial z}\right|}, \\
& \widetilde{C}_{t}^{i}(\boldsymbol{x}):=\frac{1}{\left|\frac{\partial u_{m}^{M}}{\partial i}\right|} \quad i=x, y .
\end{aligned}
$$

Then, we evaluated these quantities along the three segments $\overline{A B}, \overline{A C}$ and $\overline{A D}$, see Fig. 4. Thus, we took as an estimation of the conduction velocities $C_{f, m}$ and $C_{t, m}$ provided by the monodomain problem the mean value of these quantities,

$$
C_{f, m}=\frac{1}{N_{r}} \sum_{\boldsymbol{x}_{i} \in \overline{A D}} \widetilde{C}_{f}\left(\boldsymbol{x}_{i}\right),
$$




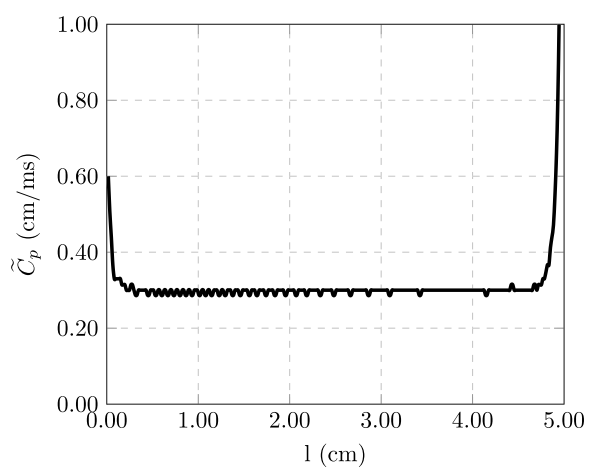

Fig. 5. Conduction velocity $\widetilde{C}_{p}$ in the Purkinje fibers as a function of the local coordinate $l$ estimated from the solution of the monodomain problem. Test for the estimation of the conduction velocity.

$$
C_{t, m}=\frac{1}{2}\left(\frac{1}{N_{x}} \sum_{\boldsymbol{x}_{i} \in \overline{A C}} \widetilde{C}_{t}^{x}\left(\boldsymbol{x}_{i}\right)+\frac{1}{N_{y}} \sum_{\boldsymbol{x}_{i} \in \overline{A B}} \widetilde{C}_{t}^{y}\left(\boldsymbol{x}_{i}\right)\right)
$$

where $N_{r}, N_{x}$ and $N_{y}$ are the numbers of points in $\overline{A D}, \overline{A C}$ and $\overline{A B}$, respectively. The conduction velocities found with these estimates were then used in the eikonal equation (2)-(3). In particular, we used $C_{f}=C_{f, m}$ and $k=C_{t, m} / C_{f, m}$. Referring to the data reported in Table 1 , we found $C_{f}=0.067 \mathrm{~cm} / \mathrm{ms}$ and $k=0.43$.

For the Purkinje network we proceeded in a similar way. In particular, we considered the propagation of a wavefront in a single Purkinje fiber of length $5 \mathrm{~cm}$ by solving the one-dimensional monodomain problem. We applied at the left boundary a current strong enough to trigger the excitation of a Purkinje cell, whereas on the right boundary a homogeneous Neumann condition. This allowed to obtain the activation time $u_{p}^{M}(\boldsymbol{x})$. Since in this case we had only one direction of propagation, we estimated the conduction velocity in the single Purkinje fiber as follows

$$
C_{p, m}=\frac{1}{N_{p}} \sum_{i=1}^{N_{p}} \widetilde{C}_{p}\left(\boldsymbol{x}_{i}\right)
$$

where $N_{p}$ is the number of nodes of the mesh discretizing the Purkinje fiber and $\widetilde{C}_{p}$ is given by

$$
\widetilde{C}_{p}(\boldsymbol{x})=\frac{1}{\left|\frac{\partial u_{p}^{M}}{\partial s}\right|} .
$$

In Fig. 5 (right) we depict the evolution of $\widetilde{C}_{p}$ in the Purkinje fiber. In particular, we used a value $\sigma_{p}$ (see Table 1 ) which allowed us to obtain a physiological value of the conduction velocity [25]. We observe that, far from the boundaries, the conduction velocity was almost constant and equal to $0.3 \mathrm{~cm} / \mathrm{ms}$, whereas, near to the boundaries, the wavefront interactions resulted in a non-constant conduction velocity. We thus used the estimated value $C_{p, m}$ as conduction velocity $C_{p}$ in the $1 \mathrm{D}$ eikonal problem (8).

For the computations we have used as discretization steps $h_{m}=0.001 \mathrm{~cm}$ for the cuboid, leading to 200000 nodes and 1.1 million tetrahedral elements, and $h_{p}=0.0165 \mathrm{~cm}$ for the Purkinje fiber. The value of $h_{p}$ was chosen equal to the characteristic length of a Purkinje cell [36].

\subsection{Numerical test in a cuboid}

In this section we report the results of a test in a cuboid for a comparison of the EE and MM coupling strategies in the case of orthodromic propagation. The myocardial geometry was the same considered in the previous section (see Fig. 3), whereas for the Purkinje fibers we considered a simple network characterized by three segments and one bifurcation point. This network lied on one side of the cuboid domain, similar to physiological situation where the Purkinje fibers are located just beneath the endocardium, see Fig. 6. The signal enters from the AV node, represented by $\boldsymbol{s}_{0}$ in Fig. 6, left, and then reaches the PMJ $\boldsymbol{s}_{1}$ and $\boldsymbol{s}_{2}$. Through these two PMJs, the signal enters the myocardium.

For the comparison, we first computed the activation maps, which are represented in Fig. 6, right. We notice that the MM and EE strategies describe a similar activation pattern in both the Purkinje network and in the myocardium. To examine further in detail the activation pattern, we also computed the cumulative percentage of activated tissue, which is depicted in Fig. 7. Note that the slope of this quantity gives us useful information about the propagation velocity of the wavefront in both domains. We begin by analyzing the Purkinje network. In Fig. 7 (left) the percentage of activated tissue in $S_{1}$ and then $S_{2}$ is represented. In particular, in the Purkinje network the EE model results in a constant conduction velocity through the 

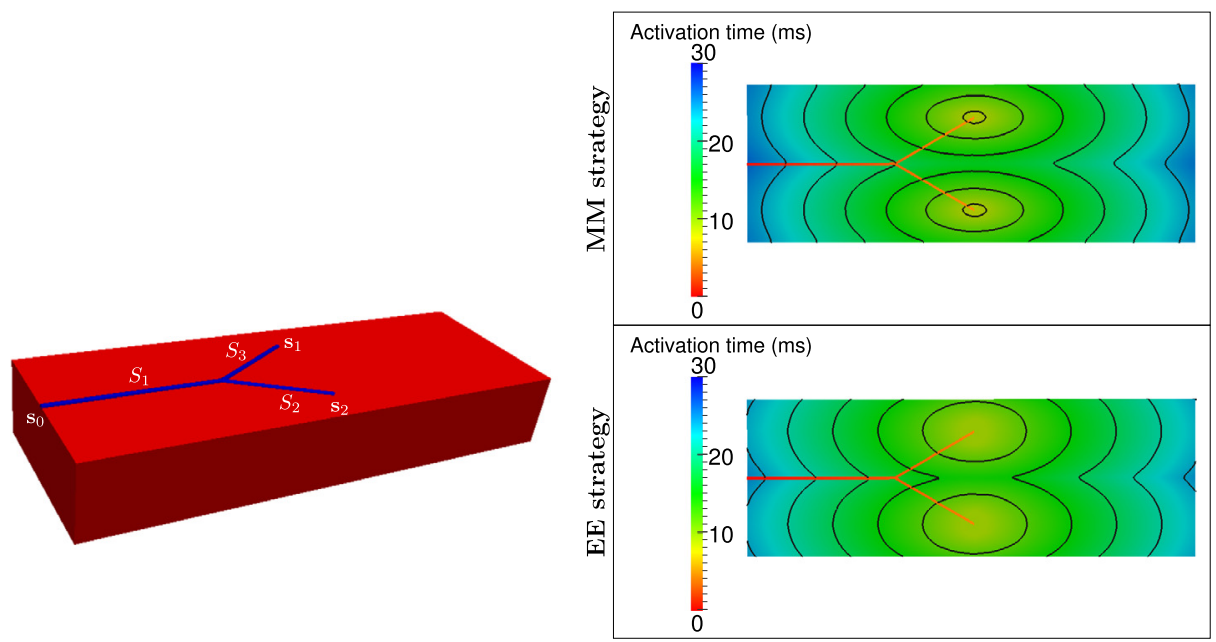

Fig. 6. Representation of the myocardium and Purkinje network domains (left), and activation maps in the case of MM (top, right) and EE (bottom, right) strategies. Test in the cuboid.
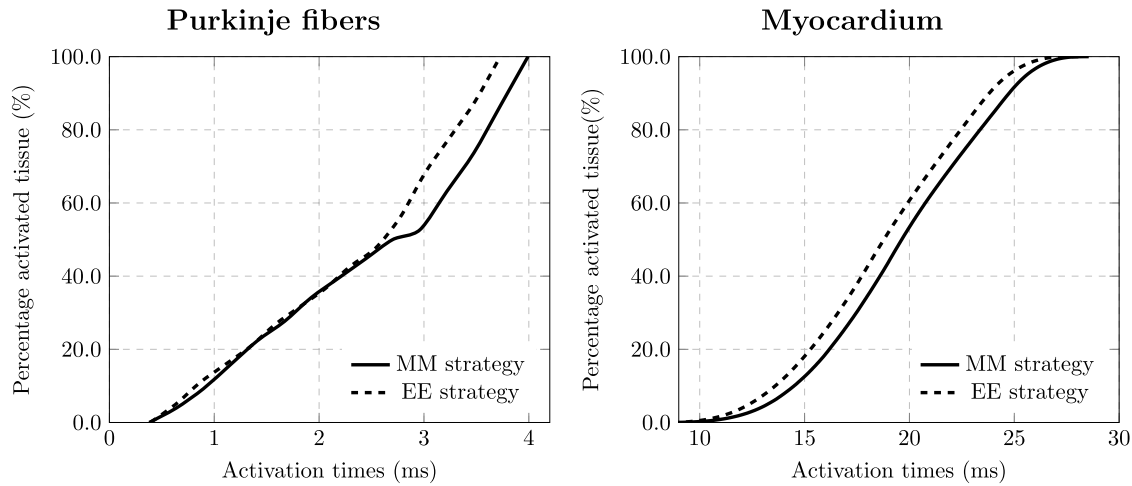

Fig. 7. Comparison of the percentage of activated tissue in the Purkinje network (left) and in the myocardium (right) for the two different coupling strategies. Test in the cuboid.

junction, whereas the MM model features a distinct discontinuity. To better investigate this phenomenon, we compute the following quantity

$$
\frac{1}{\left|\frac{\partial u_{p}^{M}}{\partial l}(\boldsymbol{x})\right|},
$$

which is an estimate of the conduction velocity in the Purkinje network provided by the monodomain problem. Notice that this quantity is different in general from the value (33), since the latter has been computed in the case of a single wavefront propagating in a single fiber.

We represent in Fig. 8 (left) the evolution of the quantity (34) in segments $S_{1}$ and $S_{2}$. In particular, we observe an initial acceleration of the signal at $\boldsymbol{s}_{0}$, reaching the value of $0.3 \mathrm{~cm} / \mathrm{ms}$, followed by a deceleration when the signal approaches the bifurcation point. After the bifurcation the signal accelerates again and the conduction velocity assumes larger values. This behavior of the conduction velocity is known as "pull and push" effect [39,40], which is due to the fact that the current just before the bifurcation point needs to increase its value in order to be able to stimulate the increased number of cells after the bifurcation. Due to energy arguments, this produces a decrement of the conduction velocity just before the bifurcation point ("pull" effect). On the contrary, the excited branches allow the increase of the value of the conduction velocity after the bifurcation point ("push" effect). Furthermore, we notice a further increment for $l>0.95$ of the conduction velocity when the signal approached the PMJ, since the resistance to the propagation decreases when the wavefront approaches the boundary. To better describe the "pull and push" effect, we also ran a simulation of a network formed only by two levels of bifurcations. In Fig. 8 (right) we report the conduction velocity as a function of the local coordinate. We observe that the signal after the "push" effect, returns to the reference value before the next "pull" effect. We notice that the "pull and push" effect can only be captured by the MM model, since in the EE model the conduction velocity is prescribed as a model parameter. 

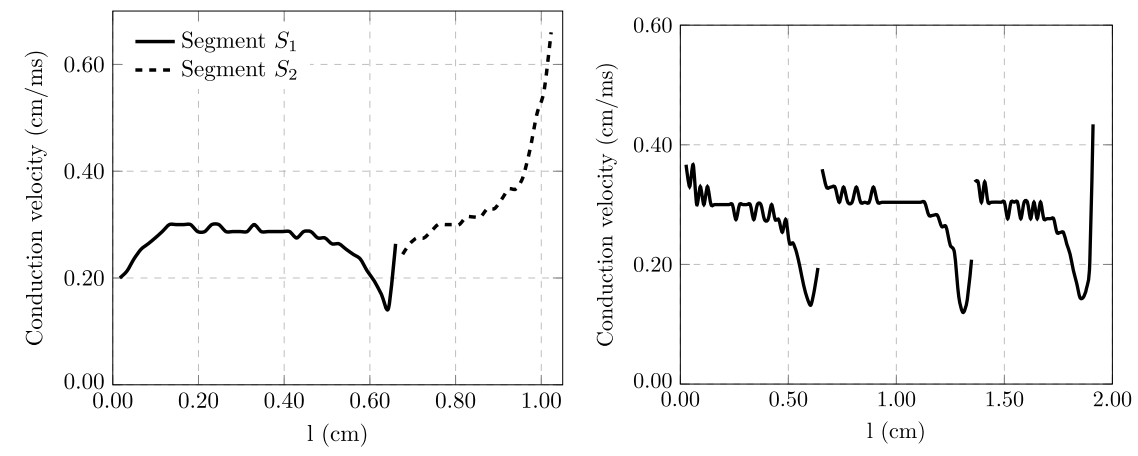

Fig. 8. Estimation of the conduction velocity in the Purkinje network as a function of the local coordinate $l$, test in the cuboid, left. Only Purkinje network with 2 levels of bifurcations, right.

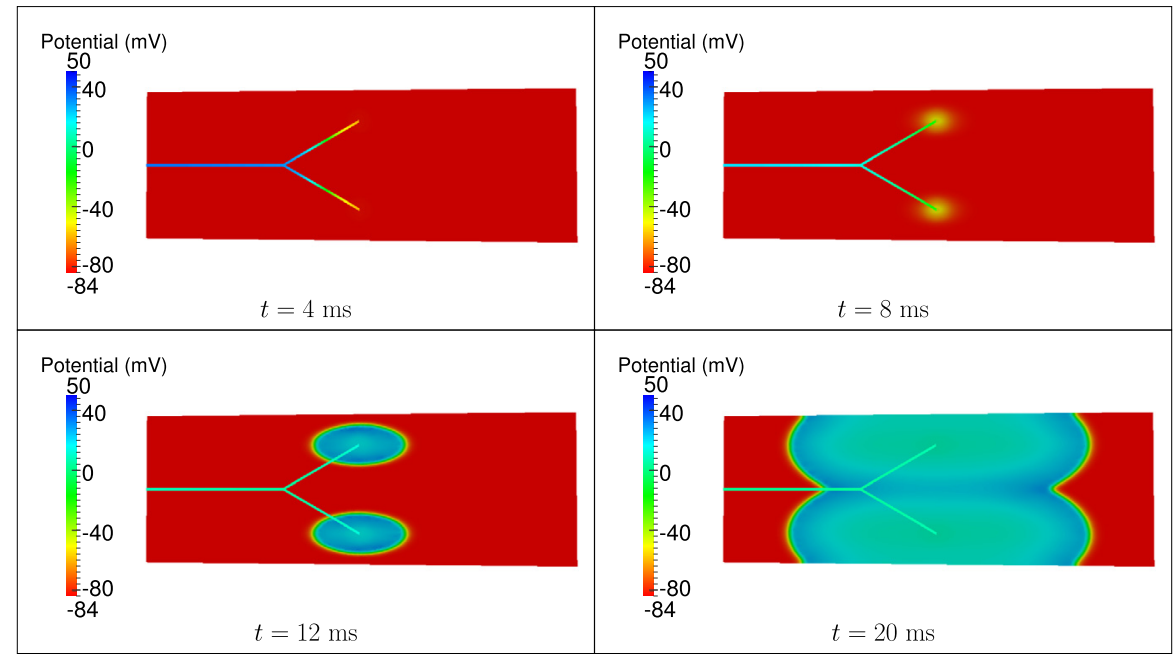

Fig. 9. Transmembrane potential at different temporal instants in the case of MM coupling. Test in the cuboid.

Coming back to Fig. 7 (right) we observe that the percentage of activated tissue in the myocardium is very similar between the EE and MM strategies. In particular, no discontinuities occur in the MM case. We notice a slow propagation velocity close to the PMJ due to the fact that at the beginning a reduced portion of 3D tissue is involved in the excitation of the neighboring non-excited tissue.

In the case of MM model, we were also able to compute the transmembrane potential in the myocardium and in the network. In particular, in Fig. 9 we report the transmembrane potential at different time instances. Notice that MM was able to model in a proper way the collision of two fronts. We also expect that the MM strategy is able to describe the delay of the propagation of the electrical signal at the PMJ due to the resistance model chosen for the latter (see (9)). Thus, in this case we did not need to impose this delay a priori as done for the EE case. To show this, we represent in Fig. 10 the transmembrane potentials computed at the PMJ $\boldsymbol{s}_{1}$ of Fig. 6 as a function of time, both for the network and the myocardium. The evolution of the simulated transmembrane potentials suggests that the delay in the orthodromic propagation corresponds in fact to the time necessary to excite the myocardial cells in the sphere $\mathcal{B}_{r}\left(\boldsymbol{s}_{1}\right)$. Additionally, we observe a good agreement with the transmembrane potentials measured at the PMJ in a canine ventricle [30], where a delay in the range $5-15 \mathrm{~ms}$ was measured.

Regarding the value of $r$ (the radius of the regions of influence centred around the PMJ), we found from the numerical experiments that a value too great is unable to activate the front in the myocardium. Conversely, if the value of $r$ is too small, we obtain an instantaneous activation of the myocardium, so that no orthodromic delay is present. A similar trend was noticed when changing the PMJ resistance $R_{P M J}$. Indeed, values too big of the PMJ resistance were unable to activate the front in the myocardium. When changing both $r$ and $R_{P M J}$ (for example by increasing the first one and decreasing the second one) we noticed a balance between the two effects and a similar sensitivity of the orthodromic delay behavior with respect to them.

Finally, we remark that the CPU time needed to solve the MM coupled problem was of course greater than the one related to the EE problem. This was due both to the fact that the latter is a steady problem, whereas the MM problem is 


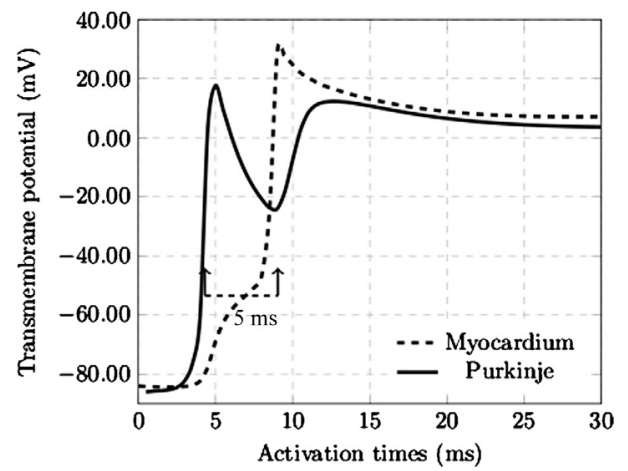

Fig. 10. Computed transmembrane potentials at the PMJ. Test in the cuboid.

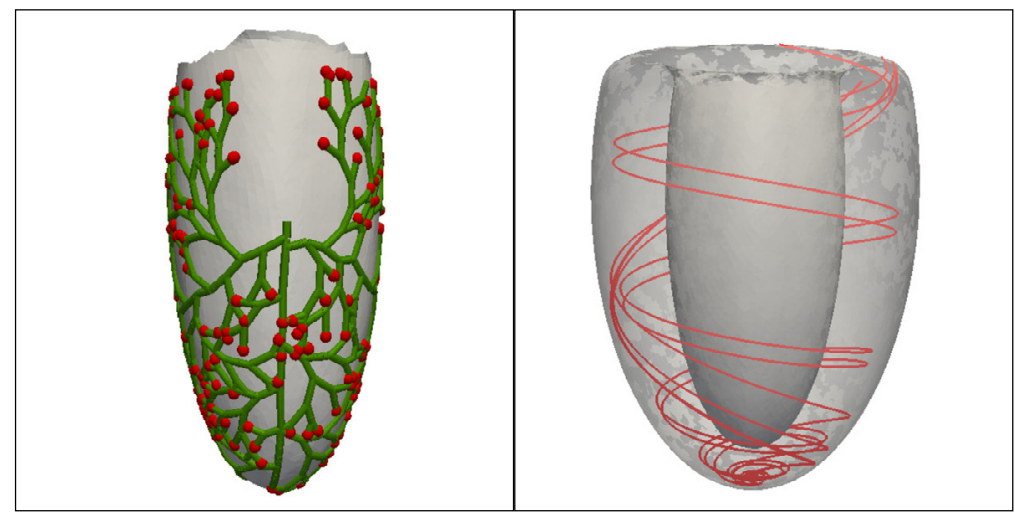

Fig. 11. Representation of the Purkinje fibers (left) and muscular fibers (right). Test in the idealized left ventricle.

unsteady, as well as due to the need of introducing subiterations (about 2-3 per time step) at each time step for the MM problem. In particular, we observe a total CPU time 20 times greater.

\subsection{Comparison in an idealized ventricle}

In this test we consider an idealized ventricular geometry given by the ellipsoidal model described in [21], where the lengths of the semi-principal axes of the inner and outer ellipsoid were $a_{x}=a_{y}=1.5 \mathrm{~cm}, a_{z}=4.4 \mathrm{~cm}$ and $b_{x}=b_{y}=$ $2.7 \mathrm{~cm}, b_{z}=5 \mathrm{~cm}$, respectively (see Fig. 11, right). To define the anisotropic tensor $D$ by (3), we set $k=0.46$ [25], and we use the analytical expression for the unit vectors tangential to the fibers proposed in [21]. The resulting mesh was composed of about 4.4 million tetrahedra and 760000 vertices, with $h_{m}=0.003 \mathrm{~cm}$.

In Fig. 11 (left) we represent the Purkinje network used in the test, consisting in 959 segments and 379 PMJ. This network has been generated by using a fractal law as proposed in [3,4] and described in [8]. The one-dimensional mesh was composed of 15000 vertices and 1400 line segments, with $h_{p}=0.0165 \mathrm{~cm}$.

The parameters used in the monodomain and eikonal problems were the same defined in the previous section and reported in Table 1, apart from the values of $r$ and $R_{P M J}$, which were set equal to $0.07 \mathrm{~cm}$ and $6000 \mathrm{kOhm}$, respectively

\subsubsection{Normal propagation}

In the first test of this section, we consider the case of a normal propagation where the unique source for the Purkinje network is the AV node and the unique sources for the myocardium were the PMJ.

For the comparison, we consider again the activation maps, represented in Fig. 12. In particular, we observe that the activation maps obtained by the two strategies in the myocardium are similar: the activation starts in the myocardium after $8 \mathrm{~ms}$ in both cases, and the last myocardial point is activated after $66 \mathrm{~ms}$ for the EE strategy and $72 \mathrm{~ms}$ for the MM strategy.

However, we notice some important differences related to the activation in the network. This is confirmed by the evolution of cumulative percentage of activated tissue, depicted in Fig. 13. In particular, Fig. 13 (left) shows that the time necessary to activate the whole network is about $16 \mathrm{~ms}$ in the case of EE model, and about $31 \mathrm{~ms}$ in the case of the MM model. This difference may be ascribed to the "pull and push" effect that introduces a delay at each branching point for the MM case. On the contrary, no substantial differences have been noted in the evolution of the percentage of activated tissue for the myocardium (Fig. 13, right), in analogy with the results of the test in the cuboid. We finally observe that in this case 


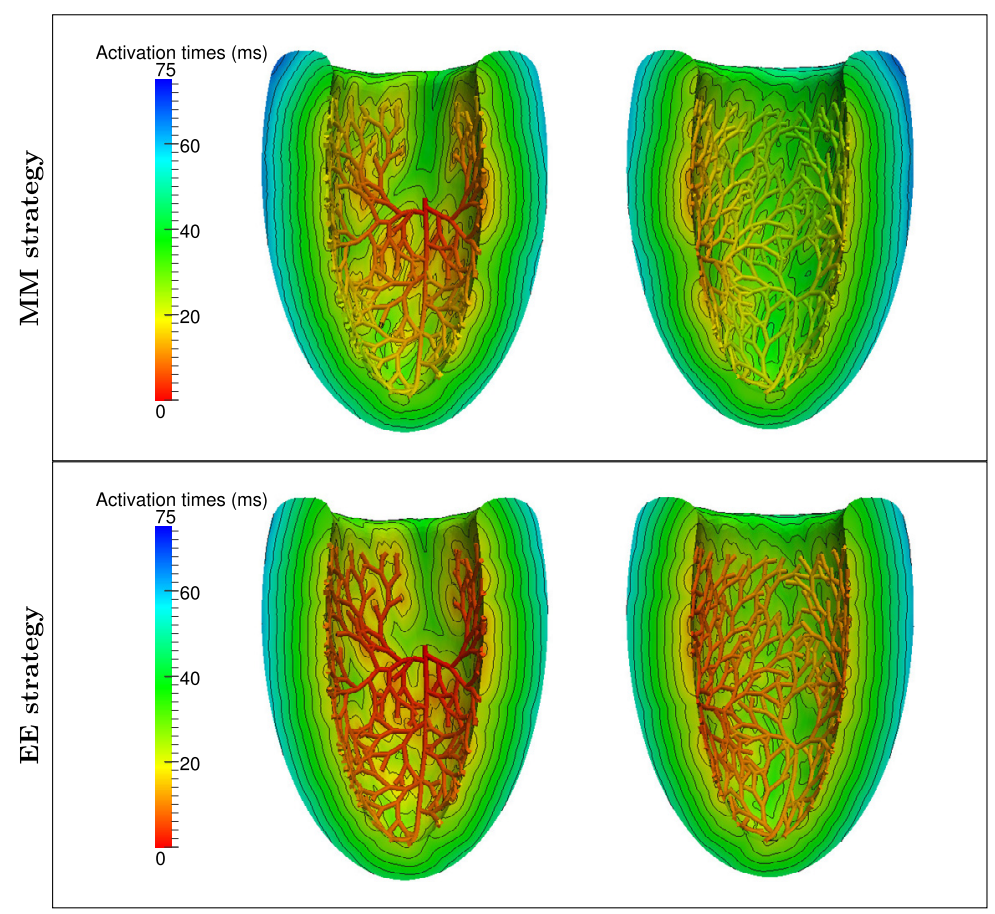

Fig. 12. Activation maps for the case of EE (bottom) and MM (top) coupling strategies. Test in the idealized left ventricle, normal propagation.
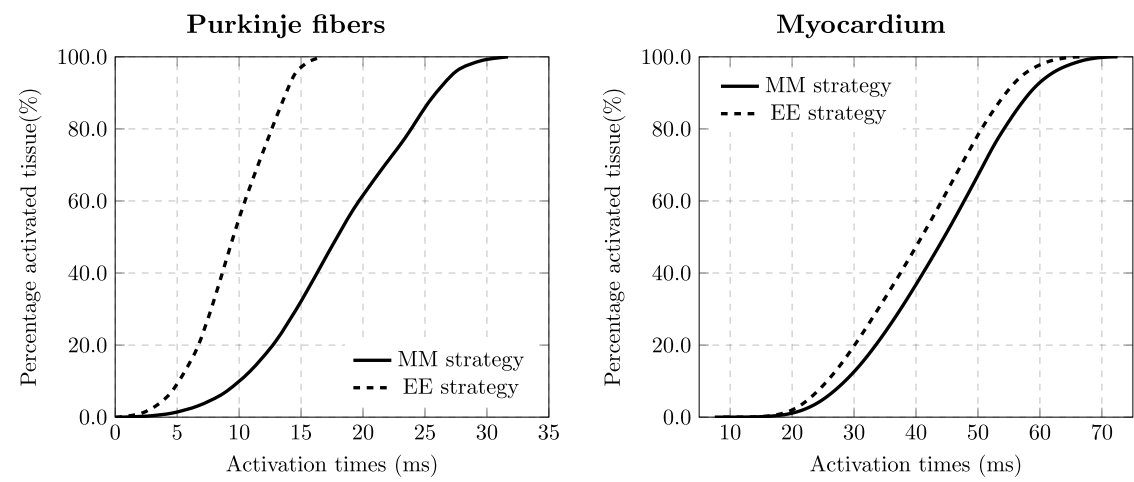

Fig. 13. Comparison of the percentage of activated tissue in the Purkinje network (left) and in the myocardium (right). Test in the idealized left ventricle, normal propagation.

the increased number of PMJ required about 4-5 iterations per time step to solve the MM coupled problem by means of Algorithm 1.

\subsubsection{Wolff-Parkinson-White syndrome}

In the second test of this section, we compare the EE and MM models for a pathological case, namely the WolffParkinson-White syndrome, which is characterized by a muscular intramyocardial source in addition to the AV node. In particular, the muscular source has been located in a point within the myocardium in the opposite region with respect to the AV node (see activation in red in Fig. 14, right). In this case, we have two fronts and thus both orthodromic and antidromic propagations. Aim of this test is to assess the suitability of Algorithm 1 in view of the solution of the MM coupled problem when multiple fronts propagate. Indeed, in this case, as highlighted in the Introduction, an explicit algorithm is not suited and we need to enforce exactly the interface conditions.

In Fig. 14 we show the activation maps from different perspectives, to highlight the two fronts. From these results, we observe of course a completely different activation with respect to the normal case. However, less pronounced differences between the EE and MM strategies can be noticed in this case. This could be ascribed to the fact that the "pull and push" effect is less pronounced in this case, since we have two fronts and thus a reduced number of consecutive bifurcation points in the network. In this case, we needed about 5-6 iterations per time step to solve the MM coupled problem. 


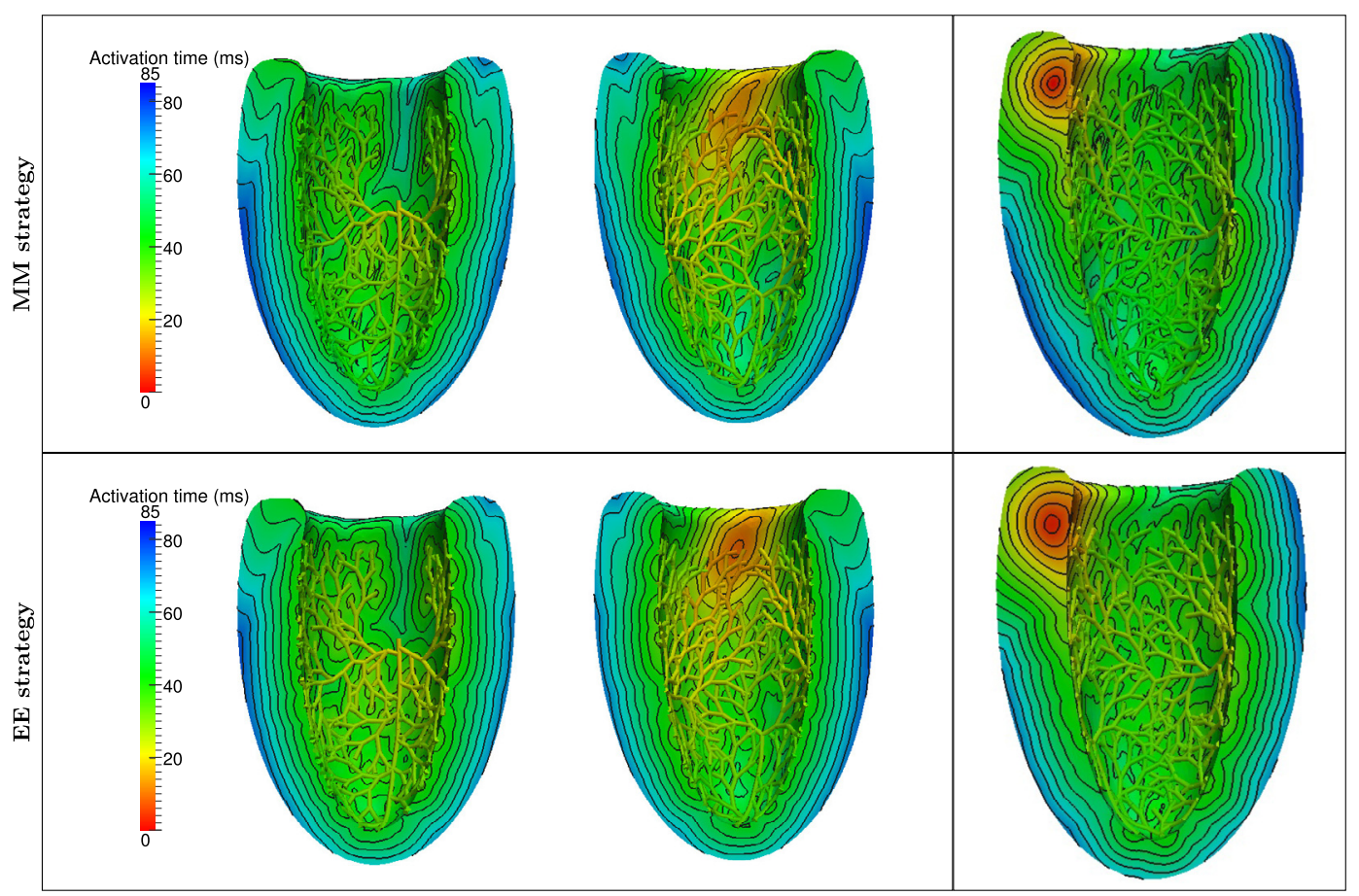

Fig. 14. Activation maps for the case of EE (bottom) and MM (top) coupling strategies. Test in the idealized left ventricle, Wolff-Parkinson-White syndrome. For each row, the left and middle figures depict two complementary views of the ventricle obtained after a cut on the sagittal plane. On the right, a view of the ventricle obtained after a cut on the coronal plane (thus perpendicular to the sagittal one) is reported. Left: front propagating from the AV node. Middle and right: front propagating from the muscular source. (For interpretation of the references to color in this figure, the reader is referred to the web version of this article.)

\subsection{Application to a realistic geometry}

In this section, we discuss the results obtained by applying the MM model to a realistic geometry, obtained by the reconstruction of the left ventricle starting from Magnetic Resonance Images (MRI). The 3D geometry has been manually segmented and it has been discretized in a tetrahedral mesh composed of about 4.7 million tetrahedra and 810000 vertices $\left(h_{m} \simeq 0.005 \mathrm{~cm}\right)$. The Purkinje network has been generated with the same fractal law used for the idealized ventricle (see Section 4.3). The corresponding one-dimensional mesh was composed of 32000 vertices and 30000 intervals, with $h_{p}=0.0165 \mathrm{~cm}$. The values of the parameters are the same reported in Table 1 , except $r=0.13 \mathrm{~cm}$ and $R_{P M J}=1000 \mathrm{kOhm}$.

In Fig. 15 we reported the activation map obtained by considering a normal propagation of the electrical signal. These results show that the MM strategy could be applied successfully also to realistic geometries. This represents a crucial step in view of solving a complete electro-mechanical simulation of a real ventricle in presence of the Purkinje network.

\section{Conclusions}

We have presented two strategies for simulating the rhythmic activation of the heart with a detailed Purkinje conduction system. In a simple model (EE model) the eikonal equation was used in the myocardium and the Purkinje system, which was then compared to solutions of the monodomain equation in both domains (MM model). The coupling conditions to be enforced between the Purkinje network and the myocardium depend on which models were adopted. For the EE model, the endpoints of the Purkinje system were connected to the myocardium with an implicit delay for the orthodromic and antidromic conduction. For the MM model, a more complex PMJ model was used, which connected the Purkinje network to the myocardium through a distributed resistance element and allowed to recover explicitly the orthodromic delay. An iterative algorithm based on fixed-point iterations was introduced to solve the MM coupled problem. Furthermore, we proved sufficient conditions for the convergence of the fixed-point iterations.

The EE and MM models have been applied first in a cuboid and then in an idealized left ventricle with healthy sinus rhythm and simulated Wolff-Parkinson-White syndrome. As indicated in previous literature, the eikonal model delivers a good approximation of the local activation time in the myocardium. However, in the Purkinje system the activation obtained by the eikonal model differs from the one obtained with the monodomain model. This is a consequence of the "pull and push" effect occurring in the Purkinje system around the branching points. We therefore found a somewhat surprising 


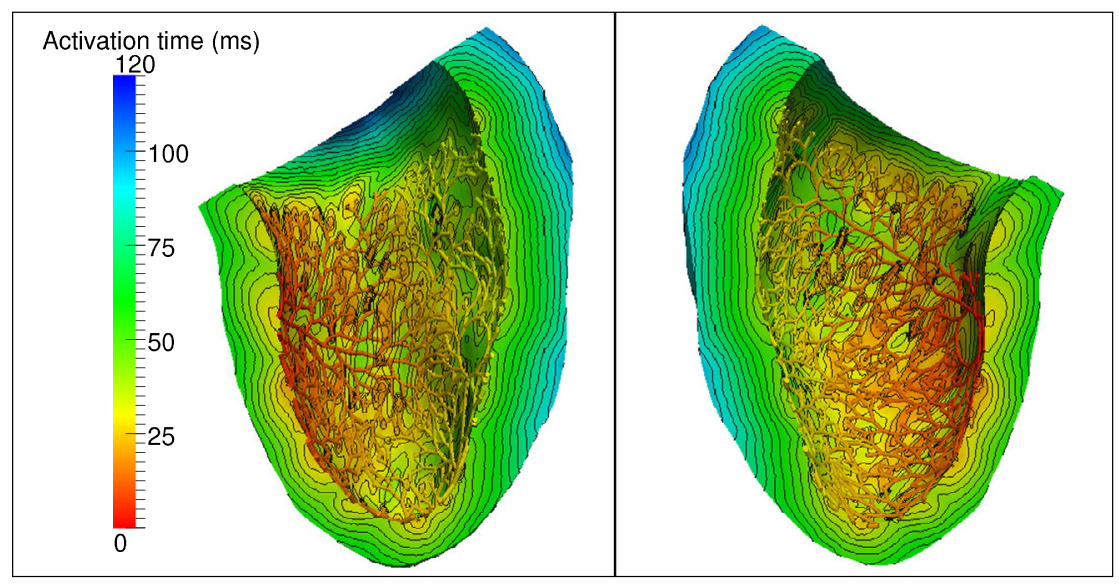

Fig. 15. Activation map obtained with MM strategy. Test in a realistic geometries.

result that seems to indicate that the use of the monodomain model is mandatory in the Purkinje network, whereas for the myocardium the eikonal approximation seems to be enough to recover accurate activation times.

The main novelties of the present work are summarized in what follows. At the best of the authors knowledge, the following points were addressed here for the first time in computational electrophysiology:

1. We proposed a numerical algorithm based on fixed-point iterations to solve the coupled problem arising by the coupling of Monodomain solvers in the Purkinje network and in the myocardium;

2. We provided a well-posedness analysis of the Monodomain-Monodomain coupled problem, which also gave sufficient conditions on the time discretization parameter in order to have convergence of the related fixed-point algorithm;

3. We provided 3D numerical simulations (both in ideal and realistic geometries) where antidromic propagations occurred, thus solving true coupled problems;

4. We were able to simulate (without an a priori imposition) the delay occurring at the PMJ, with an excellent agreement with measured data;

5. Our numerical experiments were able to reproduce the so called pull and push effect in the Purkinje network.

\section{Acknowledgements}

SP has been funded by "Fondazione Cassa di Risparmio di Trento e Rovereto" (CARITRO) within the project Numerical modeling of the electrical activity of the heart for the study of the ventricular dyssynchrony. CV and AQ has been partially supported by the Italian MIUR PRIN09 project no. 2009Y4RC3B_001. All authors declare that there exists no conflict of interest.

\section{References}

[1] R.H. Clayton, O. Bernus, E.M. Cherry, H. Dierckx, F.H. Fenton, L. Mirabella, A.V. Panfilov, F.B. Sachse, G. Seemann, H. Zhang, Models of cardiac tissue electrophysiology: progress, challenges and open questions, Prog. Biophys. Mol. Biol. 104 (1-3) (2011) $22-48$.

[2] S. Abboud, O. Berenfeld, D. Sadeh, Simulation of high-resolution QRS complex using a ventricular model with a fractal conduction system. Effects of ischemia on high-frequency QRS potentials, Circ. Res. 68 (6) (1991) 1751-1760.

[3] T. Ijiri, T. Ashihara, T. Yamaguchi, K. Takayama, T. Igarashi, T. Shimada, T. Namba, R. Haraguchi, K. Nakazawa, A procedural method for modeling the Purkinje fibers of the heart, J. Physiol. Sci. 58 (7) (2008) 90-100.

[4] R. Sebastian, V. Zimmerman, D. Romero, A.F. Frangi, Construction of a computational anatomical model of the perpheral cardiac conduction system, IEEE Trans. Biomed. Eng. 58 (12) (2011) 90-100.

[5] C. Vergara, S. Palamara, D. Catanzariti, C. Pangrazzi, F. Nobile, M. Centonze, E. Faggiano, M. Maines, A. Quarteroni, G. Vergara, Patient-specific generation of the Purkinje network driven by clinical measurements of a normal propagation, Med. Biol. Eng. Comput. 52 (10) (2014) $813-826$.

[6] S. Palamara, C. Vergara, D. Catanzariti, E. Faggiano, Pangrazzi, M. Centonze, F. Nobile, M. Maines, A. Quarteroni, Computational generation of the Purkinje network driven by clinical measurements: the case of pathological propagations, Int. J. Numer. Methods Biomed. Eng. 30 (2014) $1558-1577$.

[7] E. Behradfar, A. Nygren, E.J. Vigmond, The role of Purkinje-myocardial coupling during ventricular arrhythmia: a modeling study, PLoS ONE 9 (2) (2014) e88000.

[8] S. Palamara, C. Vergara, E. Faggiano, F. Nobile, An effective algorithm for the generation of patient-specific Purkinje networks in computational electrocardiology, J. Comput. Phys. 283 (2015) 495-517.

[9] E.J. Vigmond, C. Clements, Construction of a computer model to investigate sawtooth effects in the Purkinje system, IEEE Trans. Biomed. Eng. 54 (3) (2007) 389-399.

[10] D. Romero, R. Sebastian, B.H. Bijnens, V. Zimmerman, P.M. Boyle, E.J. Vigmond, A.F. Frangi, Effects of the Purkinje system and cardiac geometry on biventricular pacing: a model study, Ann. Biomed. Eng. 38 (4) (2010) 1388-1398.

[11] R.M. Bordas, K. Gillow, D. Gavaghan, B. Rodríguez, D. Kay, A bidomain model of the ventricular specialized conduction system of the heart, SIAM J. Appl. Math. 72 (5) (2012) 1618-1643.

[12] T. Washio, J. Okada, T. Hisada, A parallel multilevel technique for solving the bidomain equation on a human heart with Purkinje fibers and a torso model, SIAM J. Sci. Comput. 30 (6) (2008) 2855-2881. 
[13] C. Tobon-Gomez, N. Duchateau, R. Sebastian, S. Marchesseau, O. Camara, E. Donal, M. De Craene, A. Pashaei, J. Relan, M. Steghofer, P. Lamata, H. Delingette, S. Duckett, M. Garreau, A. Hernandez, K.S. Rhode, M. Sermesant, N. Ayache, C. Leclercq, R. Razavi, N.P. Smith, A.F. Frangi, Understanding the mechanisms amenable to CRT response: from pre-operative multimodal image data to patient-specific computational models, Med. Biol. Eng. Comput. 51 (11) (2013) 1235-1250.

[14] J.P. Keener, K. Bogar, A numerical method for the solution of the bidomain equations in cardiac tissue, Chaos 8 (1) (1998) $234-241$.

[15] E.J. Vigmond, F. Aguel, N.A. Trayanova, Computational techniques for solving the bidomain equations in three dimensions, IEEE Trans. Biomed. Eng. 49 (11) (2002) 1260-1269.

[16] P. Colli Franzone, L.F. Pavarino, A parallel solver for reaction-diffusion systems in computational electrocardiology, Math. Models Methods Appl. Sci. 14 (06) (2004) 883-911.

[17] P. Colli Franzone, L.F. Pavarino, B. Taccardi, Simulating patterns of excitation, repolarization and action potential duration with cardiac bidomain and monodomain models, Math. Biosci. 197 (1) (2005) 35-66.

[18] M. Potse, B. Dubé, J. Richer, A. Vinet, R.M. Gulrajani, A comparison of monodomain and bidomain reaction-diffusion models for action potential propagation in the human heart, IEEE Trans. Biomed. Eng. 53 (12) (2006) 2425-2435.

[19] J.P. Keener, An eikonal-curvature equation for action potential propagation in myocardium, J. Math. Biol. 29 (7) (1991) 629-651.

[20] P. Colli Franzone, L. Guerri, Spreading excitation in 3-D models of the anisotropic cardiac tissue, I. Validation of the Eikonal model, Math. Biosci. 113 (1993) 145-209.

[21] P. Colli Franzone, L. Guerri, M. Pennacchio, B. Taccardi, Spreading excitation in 3-D models of the anisotropic cardiac tissue, II. Effects of the fiber architecture and ventricular geometry, Math. Biosci. 147 (1998) 131-171.

[22] J.A. Sethian, Fast marching methods, SIAM Rev. 41 (2) (1999) 199-235.

[23] J.P. Keener, J. Sneyd, Mathematical Physiology. Interdisciplinary Applied Mathematics, Springer, 1998.

[24] M. Lange, S. Palamara, T. Lassila, C. Vergara, A. Quarteroni, A.F. Frangi, Verification and performance comparison of numerical solutions of electrophysiology on Purkinje networks with the CPU and GPU, MOX-Report n. 45-2015, Department of Mathematics, Politecnico di Milano, Italy, 2015.

[25] B. Kerckoffs, O.P. Faris, P.H. Boveenderd, F.W. Prinzen, K. Smits, T. Arts, Timing of depolarization and contraction in the paced canine left ventricle, J. Cardiovasc. Electrophysiol. 14 (2003) S188-S195.

[26] O. Berenfeld, J. Jalife, Purkinje-muscle reentry as a mechanism of polymorphic ventricular arrhythmias in a 3-dimensional model of the ventricles, Circ. Res. 2 (10) (1998) 1063-1077.

[27] D.J. Huelsing, K.W. Spitzer, J.M. Cordeiro, A.E. Pollard, Conduction between isolated rabbit Purkinje and ventricular myocytes coupled by a variable resistance, Am. J. Physiol., Heart Circ. Physiol. 274 (4) (1998) H1163-H1173.

[28] J. Tranum-Jensen, A.A. Wilde, J.T. Vermeulen, M.J. Janse, Morphology of electrophysiologically identified junctions between Purkinje fibers and ventricular muscle in rabbit and pig hearts, Circ. Res. 69 (2) (1991) 429-437.

[29] O.V. Aslanidi, P. Stewart, M.R. Boyett, H. Zhang, Optimal velocity and safety of discontinuous conduction through the heterogeneous Purkinje-ventricular junction, Biophys. J. 97 (1) (2009) 20-39.

[30] C. Mendez, W.J. Mueller, X. Urguiaga, Propagation of impulses across the Purkinje fiber-muscle junctions in the dog heart, Circ. Res. 26 (2) (1970) $135-150$.

[31] S. Salsa, Partial Differential Equations in Action: From Modelling to Theory, Universitext, Springer, 2008.

[32] D. Di Francesco, D. Noble, A model of cardiac electrical activity incorporating ionic pumps and concentration changes, Philos. Trans. R. Soc. B 307 (1133) (1985) 353-398.

[33] C. Luo, Y. Rudy, A model of the ventricular cardiac action potential. Depolarization, repolarization, and their interaction, Circ. Res. 68 (6) (1991) $1501-1526$.

[34] E. Konukoglu, M. Sermesant, O. Clatz, J.M. Peyrat, H. Delingette, N. Ayache, A recursive anisotropic fast marching approach to reaction diffusion equation: application to tumor growth modeling, in: IPMI 2007, in: Lect. Notes Comput. Sci., vol. 4584, Springer-Verlag, Berlin, Heidelberg, 2007.

[35] S.A. Niederer, E. Kerfoot, A.P. Benson, M.O. Bernabeu, O. Bernus, C. Bradley, E.M. Cherry, R. Clayton, F.H. Fenton, A. Garny, et al., Verification of cardiac tissue electrophysiology simulators using an n-version benchmark, Philos. Trans. R. Soc. A 369 (1954) (2011) 4331-4351.

[36] T. Stankovičová, V. Bito, F. Heinzel, K. Mubagwa, K.R. Sipido, Isolation and morphology of single Purkinje cells from the porcine heart, Gen. Physiol. Biophys. 22 (2003) 329-340.

[37] A. Martinez-Palomo, J. Alanis, D. Benitez, Transitional cardiac cells of the conductive system of the dog heart. Distinguishing morphological and electrophysiological features, J. Cell Biol. 47 (1) (1970) 1-17.

[38] P.M. Boyle, G.D. Veenhuyzen, E.J. Vigmond, Fusion during entrainment of orthodromic reciprocating tachycardia is enhanced for basal pacing sites but diminished when pacing near Purkinje system end points, Heart Rhythm 10 (3) (2013) 444-451.

[39] J.P. Kucera, A.G. Kléber, S. Rohr, Slow conduction in cardiac tissue, II. Effects of branching tissue geometry, Circ. Res. 83 (8) (1998) $795-805$.

[40] J.P. Kucera, Y. Rudy, Mechanistic insights into very slow conduction in branching cardiac tissue a model study, Circ. Res. 89 (9) (2001) $799-806$. 